\title{
LHC signals for singlet neutrinos from a natural warped seesaw mechanism. I
}

\author{
Kaustubh Agashe, ${ }^{*}$ Peizhi Du, ${ }^{\dagger}$ and Sungwoo Hong ${ }^{\ddagger}$ \\ Maryland Center for Fundamental Physics, Department of Physics, University of Maryland, \\ College Park, Maryland 20742, USA
}

(Received 9 June 2017; published 23 April 2018)

\begin{abstract}
Recently, it was shown in K. Agashe et al. [Phys. Rev. D 94, 013001 (2016)] that a straightforward implementation of the type I seesaw mechanism in a warped extra dimensional framework is in reality a natural realization of "inverse" seesaw; i.e., the Standard Model (SM) neutrino mass is dominantly generated by exchange of pseudo-Dirac TeV-mass SM singlet neutrinos. By the AdS/CFT correspondence, this scenario is dual to these singlet particles being composites of some new strong dynamics, along with the SM Higgs boson (and possibly the top quark), with the rest of the SM particles being mostly elementary. We study signals from production of these heavy neutrinos at the Large Hadron Collider $(\mathrm{LHC})$. We focus on the scenario where the strong sector has a global $S U(2)_{\mathrm{L}} \times S U(2)_{\mathrm{R}} \times U(1)_{\mathrm{X}}$ symmetry; such a left-right (LR) structure being motivated by consistency with the electroweak (EW) precision tests. The singlet neutrinos are charged under $S U(2)_{\mathrm{R}} \times U(1)_{\mathrm{X}}$ symmetry, thus can be produced from $W_{R}^{ \pm}$exchange, as in four-dimensional LR symmetric models. However, the direct coupling of light quarks to $W_{R}^{ \pm}$is negligible, due to $W_{R}^{ \pm}$also being composite (cf. four-dimensional LR models); nonetheless, a sizable coupling can be induced by mixings among the various types of $W^{ \pm}$bosons. Furthermore, $W_{R}^{ \pm}$decays dominantly into the singlet and composite partner of charged lepton (cf. SM lepton itself in four-dimensional LR model). This heavy charged lepton, in turn, decays into SM lepton, plus $Z$ /Higgs, thus the latter can be used for extra identification of the signal. For a benchmark scenario with $W_{R}^{ \pm}$of mass $2 \mathrm{TeV}$ and singlet neutrino of mass $750 \mathrm{GeV}$, we find that, in both the dilepton + dijet + Higgs and trilepton + Higgs channels, significant evidence can be seen at the $14 \mathrm{TeV}$ LHC for an integrated luminosity of $300 \mathrm{fb}^{-1}$ and that even discovery is possible with slightly more luminosity.
\end{abstract}

DOI: 10.1103/PhysRevD.97.075032

\section{INTRODUCTION}

The seesaw mechanism [1] is a very attractive and hence perhaps the most popular one for explaining the extreme smallness of the standard model (SM) neutrino masses relative to those of the charged fermions. The basic idea is illustrated by the following schematic formula,

$$
\text { generic seesaw: } m_{\nu} \sim \frac{m_{D}^{2}}{M_{N}} \text {, }
$$

where $m_{D}$ denotes the Dirac mass term between the SM doublet left-handed (LH) neutrino $\left(\nu_{L}\right)$ and a SM singlet

\footnotetext{
*kagashe@umd.edu

†pdu@umd.edu

"sungwoo83hong@gmail.com
}

Published by the American Physical Society under the terms of the Creative Commons Attribution 4.0 International license. Further distribution of this work must maintain attribution to the author(s) and the published article's title, journal citation, and DOI. Funded by SCOAP ${ }^{3}$. right-handed neutrino $\left(N_{R}\right.$ or simply $\left.N\right)$, induced by the vacuum expectation value (VEV) of the SM Higgs boson and $M_{N}$ is the Majorana mass term for the singlet.

However, it is perhaps fair to say that in its actual realizations, one typically ends up with a tuning of parameters (albeit not always fine-tuned, i.e., not involving large cancellations therein); here, we give some examples of this point. We focus here on obtaining the overall size of the SM neutrino mass in the observed ballpark, say, $\sim O(0.1) \mathrm{eV}$, and not on the detailed spectrum, including the mixing angles or mild hierarchy of mass differences etc., although latter is also important to realize within a model for a complete understanding of the SM neutrino sector. Then, we discuss a natural version in a warped extra dimensional model (dual to a four-dimensional framework of composite Higgs and partially composite rest of the SM) [2-4], which is the subject of further study in this paper.

In the original seesaw, the typical choice is that the above Dirac mass term between the two neutrinos is of order the Higgs VEV, $v$ (or somewhat smaller), and similarly, the Majorana mass term for singlet is close to the UV cutoff scale (denoted by $M_{\mathrm{UV}}$ ): 


$$
\begin{aligned}
& \text { high }- \text { scale seesaw: } m_{D} \lesssim v(\text { no tuning }) \\
& M_{N} \sim M_{\mathrm{UV}}(\text { no tuning, but see below!) }
\end{aligned}
$$

(Note that in the above and in what follows, $v$ can be replaced by $m_{\tau}$, i.e., the largest of the charged lepton masses without qualitative change in conclusions.). Plugging Eq. (2) into Eq. (1), this results in the SM neutrino mass being much smaller than the electroweak symmetry breaking (EWSB) scale.

However, the observed SM neutrino mass (assuming this is set by the largest of neutrino mass squared differences that have been confirmed, i.e., the atmospheric neutrino oscillations scale) requires that $M_{\mathrm{UV}}$ in Eq. (2) be actually several orders of magnitude smaller than the Planck scale:

$m_{\nu} \sim 0.1 \mathrm{eV} \Rightarrow M_{\mathrm{UV}} \sim 10^{14} \mathrm{GeV} \ll M_{\mathrm{Pl}} \sim 10^{18} \mathrm{GeV}$.

Of course, the latter hierarchy can be technically natural (i.e., radiatively stable), but the point is that realizing all this might require additional dynamics. For example, if this scale corresponds to spontaneous breaking of a gauge symmetry [as in $S U(2)_{L} \times S U(2)_{R} \times U(1)_{B-L}$ or left-right (LR) symmetric models; i.e., $N_{R}$ is part of a doublet of $S U(2)_{R}$ ] by a scalar VEV, then we have to explain why this scalar mass term is much smaller than the Planck scale.

An alternative is to set the singlet mass scale to be close to the IR (low-scale seesaw), for example, weak scale:

low/TeV-scale seesaw: $M_{N} \sim M_{\mathrm{IR}}(\sim \mathrm{TeV})$ (no tuning),

but then the tuning is transferred to the Dirac mass term instead:

$$
m_{\nu} \ll v \Rightarrow m_{D} \ll v .
$$

Finally, the so-called "inverse" seesaw [5] seeks to have natural choices for both the Dirac mass term between doublet and singlet neutrinos (i.e., $\lesssim v$ ) and the mass term for the singlet by itself (that too at the IR/weak scale). ${ }^{1}$ However, in the inverse seesaw, the singlet neutrino is a Dirac fermion, requiring introduction of another lefthanded (LH) singlet denoted by $S$ :

$$
M_{N S} \sim M_{\mathrm{IR}}(\sim \mathrm{TeV}) \text { (no tuning). }
$$

In addition, the second singlet has a small Majorana mass term denoted by $\mu$ so that the SM neutrino mass formula ends up looking like

$$
\text { inverse seesaw: } m_{\nu} \sim \frac{m_{D}^{2}}{M_{N S}^{2}} \mu \text {. }
$$

\footnotetext{
${ }^{1}$ The linear seesaw [6] has some features which are similar to the inverse seesaw.
}

Of course, tuning is then shifted to the Majorana mass term for $S$ :

$$
m_{\nu} \ll v \Rightarrow \mu \ll M_{N S}
$$

So, it seems that four-dimensional models of the seesaw might not be entirely satisfactory as far as explaining fully the smallness of the observed SM neutrino mass. Recently, it was emphasized [4] that

(i) a natural realization of the seesaw mechanism occurs in the warped extra-dimensional framework. ${ }^{2}$

This framework is dual, following the AdS/CFT correspondence, to varying degrees of compositeness of the SM particles. In a sense, this implementation actually features both high-scale and inverse seesaw mentioned above. Namely, from a bottom-up viewpoint, the SM neutrino mass is generated by exchange of pseudo-Dirac singlet states as in the inverse seesaw case. Remarkably,

(i) the smallness of the required Majorana mass term $(\mu)$ for the inverse seesaw is itself due to a high-scale seesaw.

Schematically, we have (with $M_{\mathrm{IR}} \sim \mathrm{TeV}$ as usual)

$$
\begin{aligned}
& \text { warped/composite seesaw: } m_{\nu} \sim \frac{m_{D}^{2}}{M_{\mathrm{IR}}^{2}} \mu, \\
& \mu \sim \frac{M_{\mathrm{IR}}^{2}}{M_{\mathrm{UV}}} \text { (no tuning). }
\end{aligned}
$$

Note that, even with the above nice feature, we still need (as alluded to above) the other hierarchy for getting the observed SM neutrino mass, i.e., $M_{\mathrm{UV}} \ll M_{\mathrm{Pl}}$ : this seems to be a tuning at first sight, but we will see that this is also explained in the warped/composite seesaw.

In detail, the dual CFT picture affords the most transparent understanding of this physics as follows (see more discussion in [4] and some using the five-dimensional model in Sec. II of this paper). The SM Higgs boson arises as a composite of some new strong dynamics which confines at the $\sim \mathrm{TeV}$ scale. Rest of the SM fields (i.e., all the gauge fields and fermions) start out as elementary degrees of freedom which are external to the strong dynamics, but they "mix" with appropriate composites of the latter. Thus, the actual SM particles are admixtures of the two sectors. Such "partial compositeness" of the SM fields allows them to couple to the SM Higgs, thus acquiring mass from its VEV. In particular, for the case of charged SM fermions, the picture is that external $S U(2)_{\mathrm{L}}$ doublet and singlet fermions mix separately with respective

\footnotetext{
${ }^{2}$ This model was originally proposed in references [2,3], but the basis used in this earlier work obscured the physical nature of the seesaw mechanism. Also, as already mentioned above, here we do not consider how to obtain the SM neutrino mixing angles etc., which might require flavor symmetries in this framework [3].
} 
composite ones, starting at the UV cutoff. Then, only in the far IR, i.e, at $\sim \mathrm{TeV}$ scale, these two types of composites (and hence the corresponding external fermions as well) "connect" to each other via the Higgs VEV.

For the neutrino sector, the story starts out similarly; i.e., we add to the SM lepton sector, an external (chiral) SM singlet, denoted by $N_{R}$, which mixes with an entire composite SM singlet tower from $\sim \mathrm{TeV}$ upwards. However, from then on, there is a departure in the script (vs that of charged fermions), again, kind of similarly to the usual seesaw models, but with some crucial difference as follows. Obviously, this concerns the "fate" of the external $N_{R}$ : namely, we assume that the strong dynamics in isolation preserves lepton number so that the composite singlets are purely Dirac to begin with. On the other hand, the external sector mass terms and interactions need not preserve lepton number; for example, $N_{R}$ has a Majorana mass term, $M_{N}$, which is close to the UV cutoff, say, $M_{\mathrm{Pl}}$.

However, even though lepton number is violated at the UV cutoff, we can not write down a SM neutrino mass operator at this stage, since the SM Higgs boson VEV is not "born" yet. Instead, the relevant effect of Majorana $N_{R}$ is that its coupling to strong dynamics will inject leptonnumber violation into the strong dynamics; in particular, integrating out $N_{R}$ (again, close to the UV cutoff) generates Majorana mass terms for the composite singlet states. Note that these Majorana mass terms are for the left chirality of composite, since that is the one with mass mixing term with external $N_{R}$.

So, we start seeing the "ingredients" for a inverse seesaw model, with the seeds being sown in the UV; in particular, it is the two chiralities of the composite singlet who play the role of the $N, S$ fields of the usual four-dimensional model of this type.

Thus, we naturally have

$$
\begin{aligned}
& \text { warped/composite seesaw: } \\
& \qquad M_{N S} \sim \mathrm{TeV} / \text { compositeness scale. }
\end{aligned}
$$

Moreover, as already advertised above, we have an explanation for smallness of the Majorana mass term for $S$ [i.e., $\mu$ in Eq. (7)]. Namely, for the TeV mass composites, this mass term will precisely be of the form of $\mu$ in Eq. (9) above; i.e., the " $\mathrm{TeV}$ " in the numerator there comes from the abovementioned mass mixing term (between $N_{R}$ and $\mathrm{LH}$ composite), and $M_{\mathrm{UV}}$ in the denominator is just the (Majorana) mass term for $N_{R}$ with itself. We will argue in a bit that this "effective" UV scale can actually be naturally smaller than Planck scale. The final $\operatorname{cog}$ in this wheel is the Dirac mass term for the composite singlet with the SM $S U(2)_{L}$ doublet neutrino: similarly to the case of the charged fermions, this arises from coupling of composite singlet to Higgs VEV and composite doublet, latter mixing with the external SM neutrino. Of course, one difference from charged fermion case is "absence" of external leg on the singlet side (since $N_{R}$ decoupled); so schematically, we get

$$
m_{D} \sim \sqrt{m_{\tau} v} \text { (no tuning) }
$$

i.e., with two external fermions, we would have gotten $m_{\tau}$ vs its "square root" here with only the external doublet present. ${ }^{3}$ In other words,

(i) the composite singlets act as a "bridge" between EWSB in the IR and lepton-number violation in the $\mathrm{UV}$, both of which are required in order to generate (Majorana) SM neutrino mass.

Note that plugging Eqs. (10), (9), and (11) into Eq. (7), we see that final formula looks like a high-scale seesaw, i.e., using Eq. (2) in Eq. (1). In fact, the procedure used in most of the previous literature $[2,3]$ for the computation of the SM neutrino mass in this warped extra-dimensional framework reinforces, as follows, this impression of highscale seesaw. In this five-dimensional model, we have a SM singlet propagating in the bulk, with a Higgs VEV-induced Dirac mass term with the SM lepton doublet field near the IR brane. In addition, this singlet field has a Majorana mass term on the UV brane, i.e., the bulk and IR brane preserve lepton number. In the so-called Kaluza-Klein (KK) basis for the singlet four-dimensional states, first the usual mode decomposition is performed by neglecting the above Majorana mass term for the singlet, resulting in zero and massive KK modes. The effects of the UV brane localized Majorana mass term on these modes are only subsequently taken into account, lifting the (would-be) zero mode and mixing them all up. It turns out that the exchange of only the would-be zero mode with a super-large Majorana mass term gives rise to the SM neutrino mass, which thus mimics a high-scale seesaw. However, some of us showed in [4] that this is not so in the mass basis; i.e., it is physically an inverse seesaw (as is clear from the above CFT viewpoint).

In fact, we can further "exploit" this process of communication between the UV (i.e., lepton-number violation) and IR (i.e., EWSB) as follows. Firstly, it is clear that the lepton-number violating perturbation to the strong dynamics (again, from integrating out the external Majorana singlet, $N_{R}$ ) has to be suitably renormalization group (RG) evolved from the UV scale to IR, i.e., over a large hierarchy. Assuming that the strong dynamics is approximately conformal over this hierarchy as would be needed in order to get the observed sizes of SM fermion masses, we see that this transmission can be significantly modulated by the anomalous dimensions of the operators involved. ${ }^{4}$ So, assuming sizable anomalous dimensions,

\footnotetext{
${ }^{3}$ For simplicity, we assume here similar degree of compositeness for doublet and singlet charged lepton.

${ }^{4}$ This corresponds to profiles for various modes in the extra dimensional dual.
} 
(i) the effective seesaw scale can be much smaller (or larger, depending on the sign of the anomalous dimensions) than the Planck scale:

again, heuristically speaking,

$$
\begin{aligned}
M_{\mathrm{UV}} & \sim M_{\mathrm{Pl}} \times(\text { anomalous scaling } \\
& \leftrightarrow \text { five-dimensional profiles }) \\
& \sim 10^{12} \mathrm{GeV} \text { (no tuning) }
\end{aligned}
$$

where the requirement of the "intermediate" scale in the second line corresponds to the choice of $m_{D}$ in the second line of Eq. (11), using this and Eq. (9) in Eq. (7) and finally setting $m_{\nu} \sim 0.1 \mathrm{eV}$. Just to be clear, there is no new dynamics at this scale, cf. usual, four-dimensional highscale seesaw. ${ }^{5}$ In short, we then have a fully natural seesaw model here, i.e., with no large hierarchies in any of the fundamental parameters.

Secondly, because we need the message of leptonnumber violation to be brought down to the $\mathrm{TeV}$ scale by particles beyond the SM, i.e., the composite singlets, we are obviously able to

(i) probe the mechanism of generation of SM neutrino mass, namely, by producing the lightest of these messengers at the Large Hadron Collider (LHC)/ future colliders (unlike the case of high-scale seesaw). Of course, this is a feature in general of inverse seesaw models so that such signals have been studied before $[7,8]$, but (as we will show here) the compositeness of the singlets make a difference!

In a series of papers (this being the first), we initiate the study of LHC signals for the $\sim \mathrm{TeV}$ mass singlets in the natural realization of (inverse) seesaw in this warped/ composite Higgs setting. We begin here by focusing on a specific but well-motivated model within the above framework. Namely,

(i) we assume that the strong dynamics has a global symmetry (in the EW sector) which contains $S U(2)_{\mathrm{L}} \times S U(2)_{\mathrm{R}} \times U(1)_{\mathrm{X}}$, of which the SM subgroup, i.e., $S U(2)_{\mathrm{L}} \times U(1)_{\mathrm{Y}}$, is gauged by external fields [with $U(1)_{\mathrm{Y}}$ being a combination of $U(1)_{\mathrm{X}}$ and the $U(1)$ contained in $\left.S U(2)_{\mathrm{R}}\right]^{6}{ }^{6}$ In the canonical case, we would identify $\mathrm{X}=(\mathrm{B}-\mathrm{L})$ as in fourdimensional LR models, but in general we could choose other representations under the extra $U(1)$. The motivation for such an extension of the EW (global) symmetry in the present context is not the one for the four-dimensional LR models, ${ }^{7}$ i.e., parity restoration at higher-energy scales, but rather that it provides a custodial symmetry for suppressing

\footnotetext{
${ }^{5}$ where, for example, this is associated with the breaking of $S U(2)_{\mathrm{R}} \times U(1)_{\mathrm{X}}$ gauge symmetry down to $U(1)_{\mathrm{Y}}$.

${ }^{6}$ The warped five-dimensional dual of this scenario is that the bulk EW gauge symmetry is extended as above and broken down to the SM subgroup on the UV brane.

${ }^{7}$ For a review, see [9].
}

the contributions of the strong dynamics to the EW precision tests, in particular, the $T$ parameter. Thus there is no elementary (i.e., external to the strong sector) $W_{R}^{ \pm}$, i.e., the charged gauge boson of the $S U(2)_{\mathrm{R}}$ group, in this model. Similarly, the combination of $U(1)_{\mathrm{B}-\mathrm{L}}$ and $U(1)$ in $S U(2)_{\mathrm{R}}$ which is orthogonal to $U(1)_{\mathrm{Y}}$-often denoted by $Z^{\prime}$-is not gauged, unlike in four-dimensional LR models; i.e., the external sector does not respect the extended EW symmetry. ${ }^{8}$ We will mostly use the elementary-composite sector picture (called two-site model [10], but augmented now by the composite singlet neutrinos) in our actual LHC signal analysis.

Even though we do not have elementary $W_{R}^{ \pm} / Z^{\prime}$ in this model, given the above global symmetry of strong dynamics, we do have

(i) composite $W_{R}^{ \pm}$and $Z^{\prime}$, ${ }^{9}$ which do couple to singlet neutrinos (cf. composites of SM gauge bosons obviously do not);

this simultaneous similarity (i.e., "existence" of $W_{R}^{ \pm}$and $Z^{\prime}$ ) and difference (their compositeness vs elementary nature) from four-dimensional LR models will be crucial to the analysis of signals for the present model.

For the lepton sector, we indeed make the canonical choice of fermion representations, but now for the composites, since it is that sector which has the $S U(2)_{R}$ symmetry, i.e.,

(i) the composite (denoted by $\psi_{e}$ ) with which the external rhs charged lepton mixes ${ }^{10}$ is part of a doublet of the (global) $S U(2)_{\mathrm{R}}$ of strong dynamics, whose other component is the composite righthanded neutrino (denoted by $\psi_{N}$ ), i.e., with which external $N_{R}$ mixes as mentioned above. ${ }^{11}$

Both $\psi$ 's have Dirac mass $\sim \mathrm{TeV}$ and are vectorlike under the SM gauge and strong dynamics global symmetries.

We begin by considering the production of $\psi_{N}$ via decays of $o n$-shell $W_{R}^{ \pm}$; again such a signal has been studied extensively in the case of usual, four-dimensional LR models [7], but the difference here is that $W_{R}^{ \pm}$is

\footnotetext{
${ }^{8}$ As a bonus, with such a symmetry structure, we automatically realize the pure Diracness of composite singlets vs large, possibly close to UV cutoff, Majorana mass term for the external singlet.

${ }^{9}$ We will denote them simply by the same symbols, since there is no chance of confusion with elementary ones in this model. Also, strictly speaking, we have to assume degeneracy of spin-1 composites here in order to classify mass eigenstates in this way: we will consider the case of nondegeneracy in a follow-up paper [11], where we will give more details of this issue.

${ }^{10}$ called "electron" here for simplicity, even though we extend this to the second and third generations also.

${ }^{11}$ In detail, one might need two such $S U(2)_{\mathrm{R}}$ doublet composites per generation-corresponding to two different five-dimensional fields-in order to obtain the correct SM charged lepton vs SM neutrino Dirac mass term, i.e., external charged lepton might actually mix with a different composite tower than the $S U(2)_{\mathrm{R}}$ partners of composite SM singlets associated with the SM neutrino mass. However, this modification does not (qualitatively) affect the present discussion.
} 
composite vs quarks inside proton being mostly elementary. So, naively, this coupling seems to be negligible (i.e., $\propto$ tiny admixture of composite in SM light quarks or the corresponding Yukawa couplings). Nonetheless, we discuss how

(i) a significant, albeit still mildly suppressed relative to $\mathrm{SM}$, light quark- $W_{R}^{ \pm}$coupling is induced.

This arises by a combination of elementary-composite mixing for $W^{ \pm}$'s corresponding to $S U(2)_{L}$ (denoted by $\left.W_{L}^{ \pm}\right)^{12}$ and composite $W_{L}^{ \pm}-W_{R}^{ \pm}$mixing induced by Higgs VEV, with the near degeneracy of these composites in a "minimal" model ${ }^{13}$ amplifying the Higgs VEV effect. ${ }^{14}$ In fact, such a mild suppression of production of $W_{R}^{ \pm}$(as compared to usual four-dimensional LR models) is perhaps "welcome" in the sense that the LHC early run 2 searches are already constraining $2 \mathrm{TeV} W_{R}^{ \pm}$in the usual case, but with compositeness, such low scale for $W_{R}$ would then (i.e., given a smaller cross section for the same mass) still be allowed. At the same time, as we will show, the coupling is sizable enough that discovery (for $2 \mathrm{TeV} W_{R}^{ \pm}$and $\sim 750 \mathrm{GeV} \psi_{e, N}{ }^{15}$ such that the above decay is allowed) by the end of run $2\left(\sim 300 \mathrm{fb}^{-1}\right)$ would be possible.

Moving onto the relevant decays of $W_{R}^{ \pm},{ }^{16}$ first of all, the largest coupling of $W_{R}^{ \pm}$involves composite partner of SM $e_{R}$ and the composite singlet neutrino, i.e., $\psi_{e}$ and $\psi_{N}$, cf. $S M e_{R}$ and singlet neutrino in the usual, four-dimensional LR case. The singlet neutrino decays predominantly (as in four-dimensional LR models) into SM doublet lepton and Higgs doublet (including physical Higgs and longitudinal $W / Z$ ) via the associated Yukawa coupling ${ }^{17}$ : the channel we will focus on here (based on smaller background, thus more visibility) is $e_{L}+W$. On the other side, $\psi_{e}$ will similarly decay: we will consider $e_{L}+Z_{\text {long }} / h$ final state here. Thus, we see that there is

(i) an "extra" Higgs/Z (vs usual, four-dimensional LR models) among the decay products of the $W_{R}^{ \pm}$, which, assuming it is tagged, can be used to reduce the SM background.

\footnotetext{
${ }^{12}$ Recall that there is no elementary gauge boson mixing directly with the composite $W_{R}^{ \pm}$.

${ }^{13}$ This is dual to the five-dimensional model with no IR branelocalized kinetic terms for bulk gauge fields.

${ }^{14}$ See Refs. [12,13] for the five-dimensional version of this effect. Also, we will consider the case of nondegenerate spin-1 composites in a follow-up paper [11].

${ }^{15} \mathrm{We}$ could contemplate even lighter singlet neutrino, but accomplishing such a hierarchy might require tuning, for example, too large brane-localized kinetic terms, given that gauge KK cannot be below $\sim 2 \mathrm{TeV}$ due to constraints from EWPT.

${ }^{16}$ Other decay channels for $W_{R}^{ \pm}$include various components of the Higgs doublet: these were studied in [13], but singlet neutrino was not included there.

${ }^{17}$ Note that this coupling is indeed small, given that it involves degree of compositeness of $S M$ (doublet) lepton, but there is not much of an "option" here in terms of decay channel, given that lepton number is (approximately) preserved.
}

Moreover, it then allows us to possibly reconstruct the full decay of $\psi_{e}$, thus determining its mass, which is same as that of $\psi_{N}$ given the $S U(2)_{R}$ symmetry. Including decays of $W$ from $\psi_{N}$, we then have

(i) two search channels, i.e., dilepton + dijet (hadronic decay of $W$ ) and trilepton (leptonic decay of $W$ ), along with Higgs/ $Z$ boson. ${ }^{18}$

We will study both of these and find them to be complementary, for example, rate is larger for the former (based simply on corresponding branching ratios of $W$ ), but so is possibly SM background, given that leptons are typically "cleaner". (Of course, for the case of hadronic decay of the $W$ from $\psi_{N}$, that side is also fully visible and hence can furnish information on $\psi_{e, N}$ masses.)

Finally, in addition to $W_{R}^{ \pm}$, we consider production of $\psi_{e, N}$ pairs from decays of on-shell $Z^{\prime}$. Once again, the "direct" coupling of quarks inside proton to $Z$ ' is negligible; however, mixing does create a larger coupling (just like for the case of $W_{R}^{ \pm}$above). Note that in usual, four-dimensional LR models, $Z^{\prime}$ is typically heavier than $W_{R}^{ \pm}$, for example, assuming both (being elementary) get their mass from some scalar VEV, just like the case of SM $W / Z$. Hence, the production cross section of $Z^{\prime}$ tends to be smaller than that of $W_{R}^{ \pm}$. However, in the seesaw model being studied here,

(i) the $W_{R}^{ \pm}$and $Z^{\prime}$ can be almost degenerate, since their masses arise from the compositeness scale so that $Z^{\prime}$ signal can be comparable to $W_{R}^{ \pm}$.

Here is the outline of the rest of this paper. We begin in Sec. II with a brief review of the basic seesaw model in the warped extra-dimensional framework and present details of the implementation in the context of the $S U(2)_{\mathrm{R}}$ extension of the SM EW symmetry mentioned above. In Sec. III, we outline the "simplified," i.e., two-site approach [10], to studying the five-dimensional model that we will employ in our actual analysis of LHC signals. We then discuss our main results, starting with production cross sections and decay branching ratios of various heavy particles in Sec. IV, followed by computations of SM background and thus the discovery potential for the new particles in Sec. V. Here, we also mention strategies (postdiscovery) for distinguishing the composite/warped seesaw model from the usual, fourdimensional LR one. We conclude and present some directions for future work in Sec. VI.

\section{FIVE-DIMENSIONAL NATURAL WARPED SEESAW MODEL}

In this section, we provide a brief review of seesaw model in five-dimensional warped extra dimensions. After

\footnotetext{
${ }^{18}$ Note that even in the usual, four-dimensional LR models, one can also get the Higgs/Z boson from singlet neutrino decay, but then we lose lepton(s), i.e., final state will be $\ell+$ Higgs $/ Z+$ MET, thereby increasing SM background (for example, SM $W+Z$ /Higgs production will then be relevant), as opposed to our case of Higgs along with di- or trileptons.
} 
discussing general features of warped seesaw, we will focus on a model with the extended bulk gauge symmetry: $S U(2)_{\mathrm{L}} \times S U(2)_{\mathrm{R}} \times U(1)_{\mathrm{X}}$. Our studies of LHC signals are performed using the simplified two-site model of the full five-dimensional warped model. Hence, our discussion about the full five-dimensional model in this section will be brief, leaving details necessary for the phenomenology to $\mathrm{Sec}$. III of the two-site model. More details about the fivedimensional results, along with their four-dimensional CFT dual description, can be found in [4].

We begin our discussion by taking usual RandallSundrum framework with all SM fermions and gauge bosons propagating the bulk of a slice of $\mathrm{AdS}_{5}$. For concreteness, we consider SM Higgs to be localized on the IR brane. The five-dimensional SM gauge singlet field, $N$, which is the analog of the right-handed neutrinos of the usual, four-dimensional seesaw models, propagates the bulk. Like all five-dimensional fermion fields, $N$ can be decomposed into both left $(L)$ and right $(R)$ chiralities (denoted by $N_{L, R}$, respectively) from the four-dimensional viewpoint. $N_{R}$ couples to $\mathrm{SM} S U(2)_{\mathrm{L}}$ lepton doublet, in particular left-handed neutrinos, and the Higgs on the IR brane with five-dimensional Yukawa coupling $y_{5 \mathrm{D}}$. We impose that the lepton number is unbroken in the bulk/IR brane, ${ }^{19}$ but is broken on the UV brane. Hence, a Majorana mass term for $N_{R}$ is forbidden in the bulk/IR brane, whereas it is allowed on the UV brane. Furthermore, we assume that the order parameter for breaking of the lepton number on the UV brane is not tuned; i.e., it is (roughly) the mass scale corresponding to its location in the extra dimension. It is then natural to include a Planck (or AdS curvature) scale-size Majorana mass term for $N_{R}$ on the UV brane. These aspects can be summarized in the following five-dimensional Lagrangian,

$$
\begin{aligned}
-\mathcal{L}_{5 \mathrm{D}} \ni y_{5 \mathrm{D}} \bar{L} \tilde{\Phi} N_{R}+c_{N} k \bar{N}_{L} N_{R} \\
\quad+\delta\left(z-z_{h}\right) \frac{1}{2} \frac{m_{N}}{k} N_{R}^{T} C N_{R}+\text { H.c. }
\end{aligned}
$$

where $\tilde{\Phi} \equiv i \sigma_{2} \Phi^{*}$ with $\Phi$ denoting Higgs doublet ( $H$ will be used for physical Higgs boson). Also, $N$ is a fivedimensional fermion field with $N_{L / R}$ being left(right)chirality of $N$. In addition, $c_{N} k$ is a five-dimensional mass parameter for $N$ (in units of the AdS curvature scale, $k$ ), $m_{N}$ is Majorana mass of $N_{R}{ }^{20}$ and $C$ denotes charge conjugation operation. $\mathrm{UV}(\mathrm{IR})$ brane is at $z=z_{h}\left(z_{v}\right)$.

The above model was studied in [2] using so-called KKbasis where KK decomposition was done without taking into account the large Majorana mass term from the beginning. The effects of the Majorana mass was added

\footnotetext{
${ }^{19}$ This symmetry should be gauged in the five-dimensional model.

${ }^{20}$ As indicated above, $m_{N} \sim O(k)$ naturally.
}

as an a posteriori process, and this leads to large Majorana masses for zero and KK modes and large mixing among all modes. Hence, although analysis using KK-basis produces correct neutrino mass formula, using a basis that is vastly different from the mass basis obscures the physical picture. In particular, the results from KK-basis naively suggest (or give the misleading impression) that the above fivedimensional warped seesaw model is indeed of type I in the sense that the SM neutrino mass is generated by the dynamical exchange of a super-heavy singlet mode, i.e., at the (effective) seesaw scale (for more discussion of this point, see [4]).

However, as shown in [4], analysis based on the mass basis, including the Majorana mass term from the beginning, reveals very different dynamical picture. The mass eigenstates of four-dimensional effective theory (after KK-decomposition) of Eq. (13) is a tower of pseudo-Dirac singlet fermions with tiny Majorana splitting. For the choice of $c_{N} \sim$ -0.3 that renders correct SM neutrino mass, dominant contributions to the SM neutrino masses come from the exchange of a few low lying mass eigenstates (cf. superheavy modes in the KK basis). Namely, the SM neutrino mass is generated not by an exchange of super-heavy Majorana singlet mode, but by exchanges of $O(\mathrm{TeV})$ pseudo-Dirac singlet modes. Therefore, the dynamical nature of the warped seesaw is inverse seesaw [5], not type I. Moreover, it is indeed a very natural realization of it, because the SM neutrino mass is obtained with all dimensionful parameters taken to be near the cutoff scale and all dimensionless parameters to be $O(1)$. This new finding, then, refocuses attention on LHC signals from the $O(\mathrm{TeV})$ scale singlet pseudo-Dirac fermions that arise in this model. Since the production and decay channels depend on details of the model, now we describe a concrete model based on the extended bulk gauge symmetry, whose simplified two-site version (presented in next section) will be used for our collider studies in Sec. V.

\section{A. Natural realization with custodial symmetry}

In order to have sizable signal production of the new particles in the five-dimensional model (i.e., the KK excitations of SM) at the LHC, a KK scale of the order $O(1) \mathrm{TeV}$ is desirable; of course naturalness of the EW scale also prefers such a low scale. However, the minimal RS model with only the SM gauge symmetry in the bulk is in tension with EW precision tests, both oblique and nonoblique (from $Z \rightarrow b \bar{b}$ coupling) corrections, and consistency requires $\mathrm{KK}$ scale of $\gtrsim O(10) \mathrm{TeV}$. This bound can be relaxed by extending the bulk $\mathrm{EW}^{21}$ gauge group to $S U(2)_{\mathrm{L}} \times S U(2)_{\mathrm{R}} \times U(1)_{\mathrm{X}}$. In particular, the extended gauge group provides custodial symmetry for both the $T$-parameter and $Z \rightarrow b \bar{b}$ coupling, and KK

\footnotetext{
${ }^{21}$ Since QCD gauge group in the bulk will not play any role in our study, we will simply drop it from hereon.
} 
scale as low as $O(1) \mathrm{TeV}$ is allowed $[14,15] .{ }^{22}$ There are also constraints from flavor/CP tests which generically require $\gtrsim O(10) \mathrm{TeV} \mathrm{KK}$ scale, but here we assume addition flavor structure (for example, flavor symmetries) in order to ameliorate those bounds [16].

On the UV brane, the gauge symmetry is broken down from $S U(2)_{\mathrm{R}} \times U(1)_{\mathrm{X}}$ to $U(1)_{\mathrm{Y}}$ by choice of boundary conditions (BC). Specifically, the gauge fields associated with the broken generators $\left(S U(2)_{\mathrm{R}} \times U(1)_{\mathrm{X}}\right) / U(1)_{\mathrm{Y}}$ will have Dirichlet BC, denoted henceforth as "-", whereas $U(1)_{Y}$ and $S U(2)_{L}$ have Neumann (+). All gauge fields are taken to be + on IR brane. In particular, only fields with $(++)$ BC have zero modes up on KK-decomposition, i.e., only gauge fields for the SM gauge group in this case. We use $W_{R}$ and $Z^{\prime}$ to denote the extra gauge fields, i.e., for charged $S U(2)_{\mathrm{R}}$ and $\left(U(1)_{\mathrm{R}} \times U(1)_{\mathrm{X}}\right) / U(1)_{\mathrm{Y}}$, respectively: these have $(-+) \mathrm{BC}$ and hence only have massive/KK modes.

Higgs field, which we choose to be localized on the IR brane, is a bidoublet of $S U(2)_{\mathrm{L}} \times S U(2)_{\mathrm{R}}$, with zero charge under $U(1)_{X}$ :

$$
\boldsymbol{\Phi} \equiv\left(i \sigma_{2} \Phi^{*}, \Phi\right) \in(2,2)_{0} .
$$

This representation results in a custodial symmetry, i.e., the Higgs VEV breaks $S U(2)_{L} \times S U(2)_{R}$ down to $S U(2)_{V}$, which suppresses contributions from the gauge sector to the $T$-parameter: note that $U(1)_{X}$ remains unbroken in this process. The Higgs VEV will also generate mixing between various modes of $W_{R}$ and $W_{L}$, an effect which can be treated perturbatively and which will be very important for LHC signals for the singlet neutrinos. We will make this point clearer in Sec. III.

Moving onto representation of fermions under the extended gauge group, first note that (just like for gauge fields) SM fermions will arise as zero modes of fivedimensional fields with $(++)$ BC. We begin with the leptons, where we choose the simplest possibility, i.e., $X$ is the same as $(B-L)$ in this sector. Thus, we take $L$, i.e., the $\mathrm{SM} S U(2)_{L}$ lepton doublet, to be a singlet of $S U(2)_{R}$, while the righthanded charged lepton (denoted by $\ell$ ) is promoted to be a doublet of $S U(2)_{R}$, denoted by $L_{R}$ [as in the canonical, fourdimensional (gauged) left-right (LR) symmetric models]:

$$
L \in(2,1)_{-\frac{1}{2}} \quad L_{R}, \tilde{L}_{R} \in(1,2)_{-\frac{1}{2}} .
$$

where numbers in the parenthesis denote representation under $S U(2)_{\mathrm{L}}$ and $S U(2)_{\mathrm{R}}$, while representation of $U(1)_{X}$ is shown as a subscript [we will explain momentarily why there are two

\footnotetext{
${ }^{22}$ In fact, even with the extended bulk gauge group, KK scale is constrained generically to be $\gtrsim O(3) \mathrm{TeV}$. Thus, special regions of parameter space and/or additional contributions to these observables (perhaps from further model building) will be needed in order to have $\mathrm{KK}$ scale as low as $O(1) \mathrm{TeV}$. Given that resonances with mass $O(3) \mathrm{TeV}$ or heavier is slightly beyond the LHC reach, having new colliders with higher energy reach are required and hence motivated for a better test.
}

$S U(2)_{R}$ doublets]. Remarkably, akin to usual, fourdimensional LR symmetric models, we see that the $S U(2)_{R}$ partner of $\ell$ has the characteristics similar to that of the $N$ field mentioned above in the context of the general seesaw model; i.e., (i) it is a singlet under the SM gauge group; (ii) it has a Yukawa coupling with lepton doublet on the IR brane and (iii) a Majorana mass term for it can be written only on the UV brane, since that is the only location where $S U(2)_{R} \times U(1)_{X}$ (under which it is charged) is broken. As a byproduct, such a choice gives rise to a way to produce the partner of $\ell$ via decay of $W_{R}$, which has similar features as our signal process.

In more detail, note that we will actually need two $S U(2)_{\mathrm{R}}$ lepton doublets, namely,

$\tilde{L}_{R}=\left(\begin{array}{l}N(++) \rightarrow(-+) \\ \tilde{\ell}(-+)\end{array}\right)_{R} \quad L_{R}=\left(\begin{array}{l}\tilde{N}(-+) \\ \ell(++)\end{array}\right)_{R}$.

Here the SM lepton $(\ell)$ is obtained as the zero mode from the second multiplet above, i.e., with $(++)$ BC; its $S U(2)_{R}$ partner (denoted by $\tilde{N}$ ) is chosen to have '-' boundary condition on the UV brane (thus having no zero mode at all): this is consistent with the bulk gauge symmetry since $S U(2)_{R} \times U(1)_{X}$ is broken on the UV brane to $U(1)_{Y}$ (i.e. different BC's for two components of doublet are allowed), while this symmetry is unbroken on IR brane (i.e. it should be same BC for both fields, which is + in this case). Note that $\tilde{N}$ then plays no role in the seesaw for the SM neutrino mass since its BC on UV being '-' it does not acquire Majorana mass. In addition, by making its mass to be larger than half of KK gauge mass, its production via the decay of KK gauge boson will be suppressed. For this reason, we will drop $\tilde{N}$ from now on. On the other hand, the BC's are "switched" in the first doublet, i.e., $\tilde{\ell}$ has no zero mode, whereas the $N$ here will be driving the SM neutrino mass seesaw (thus will be denoted as the singlet neutrino henceforth). Note that $N$ has (++) BC to "begin with," but adding a UV brane localized Majorana mass term "repels" $N$ profile away from UV brane, resulting in effective boundary condition of the form $(-+)$ and hence removing the corresponding zero mode.

The simple reason for having two $S U(2)_{R}$ doublets, instead of housing $N$ and SM right-handed lepton in a single $S U(2)_{R}$ doublet, is the following. The $N$ and SM right-handed lepton, i.e., $\ell$, fields should have different five-dimensional bulk mass parameters in order to produce correct masses for charged lepton and neutrino [2]; i.e., we require $c<-0.5$ for the field giving charged lepton zero mode so that this mode is localized near the UV brane, ${ }^{23}$ thus giving the observed charged lepton mass, whereas we need $c \sim-0.3$ for $N$ (as mentioned above), i.e., the wouldbe zero mode should be peaked near the IR brane instead.

\footnotetext{
${ }^{23}$ Such a profile also needs to be chosen for the $L$ zero mode.
} 


$$
\begin{aligned}
& S U(2)_{L} \times S U(2)_{R} \times U(1)_{X} \\
& \begin{array}{lll}
\Downarrow & \quad S U(2)_{L} \times S U(2)_{R} \times U(1)_{X} & S U(2)_{L} \times S U(2)_{R} \times U(1)_{X}
\end{array}
\end{aligned}
$$

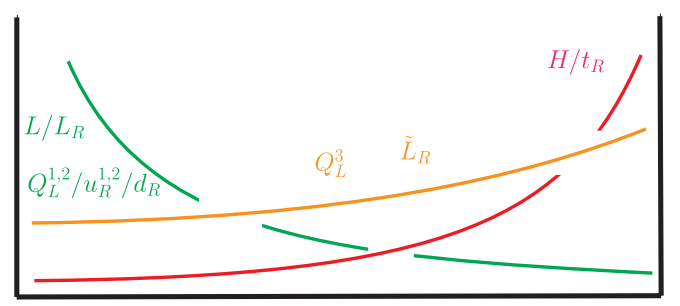

FIG. 1. RS model with extended bulk gauge symmetry $S U(2)_{\mathrm{L}} \times S U(2)_{\mathrm{R}} \times U(1)_{\mathrm{X}}$ and singlet neutrino. Gauge symmetries together with its breaking pattern are shown on the relevant position along the extra-dimension. The position of the fields shows where the zero-mode profile of the corresponding five-dimensional fields is localized. Fields with (close to) flat zero-mode profile are located in the middle of the bulk.

However, by $S U(2)_{R}$-invariance, fields in a doublet should have a common five-dimensional mass parameter. Thus, we need to "split" the SM charged lepton and singlet neutrino multiplets as shown above.

Following [15], i.e., in order to suppress corrections to the $Z b \bar{b}$ coupling, we choose the representations of the quarks to be somewhat nonminimal as follows.

$$
Q_{L} \in(2,2)_{\frac{2}{3}} \quad u_{R} \in(1,1)_{\frac{2}{3}} \quad d_{R} \in(1,3)_{\frac{2}{3}} .
$$

Here, $Q_{L}$ denotes the SM left-handed quarks doublet and $u_{R}, d_{R}$ are the $S U(2)_{\mathrm{L}}$ singlets. For the "extra" fields in $S U(2)_{\mathrm{R}}$ doublet or triplet representations above, we take Dirichlet-Neumann $(-+)$ boundary condition in order to remove the zero mode (just like was done for leptons above). As usual, $t_{R}$ zero mode is taken to be localized near the IR brane, while $(t, b)_{L}$, i.e., $Q_{L}^{3}$, has a (roughly) flat profile and rest of the quarks are peaked near the UV brane (just like the SM leptons).

We mentioned that the spectrum of $N$ in fourdimensional effective theory is a tower of pseudo-Dirac fermions. Since the Majorana splitting $[O(\mathrm{MeV})]$ for these pseudo-Dirac pairs is very tiny comparing to its Dirac mass $[O(\mathrm{TeV})]$, we are unlikely to be able to probe any effects from such Majorana splitting. Moreover, as far as investigating the discovery potential of the lightest pseudo-Dirac singlet mode is concerned, the existence of tiny Majorana splitting will not make any difference. For this reason and for simplicity, in our collider study, we ignore Majorana splitting and treat $N$ as pure Dirac with $(-+)$ boundary condition, which will have the same mass as its $S U(2)_{\mathrm{R}}$ partner $\tilde{\ell}$.

The five-dimensional fields discussed thus far are summarized in Fig. 1. The position of the fields shows where the zero-mode profile of the corresponding fivedimensional fields is localized. Fields that are closer to the UV (IR) brane signifies that their zero-mode profiles are peaked near the UV (IR) brane. For fields with (close to) flat zero-mode profile, they are located in the middle of the bulk.

\section{B. Couplings of KK modes}

The couplings among various four-dimensional particles are proportional to the overlap of their respective profiles in the extra dimension. Now the light quarks are localized near the UV brane, while the KK modes are near the IR brane. However, the nonzero (Neumann BC) profile of KK of SM gauge bosons at the UV brane does induce a significant coupling to the light quarks. On the other hand, KK $W_{R}^{ \pm}$and $Z^{\prime}$ vanish at the UV brane (Dirichlet BC), rendering such a coupling to be negligible. Nonetheless, as we discuss in Sec. III, EWSB mixing among KK $W_{L}^{ \pm}$and $W_{R}^{ \pm}$does induces a sizable coupling of $\mathrm{KK} W_{R}^{ \pm}$to light quarks provided there is degeneracy between $\mathrm{KK} W_{R}^{ \pm}$and $\mathrm{KK} W_{L}^{ \pm}$, similarly $\mathrm{KK} Z$ and $\mathrm{KK} Z^{\prime}$. This coupling can then be used in production of $\mathrm{KK} W_{R}^{ \pm}$and $Z^{\prime}$. Once produced, their decay is dominantly to modes localized near IR brane such as (light) KK fermions and/or top quark/Higgs boson, since those couplings are the largest.

\section{Spectrum of KK modes}

Mass of KK gauge boson is dictated by boundary condition of the corresponding five-dimensional gauge field. The mass of the first KK mode of gauge fields with $(++)$ boundary condition is typically $O(1) \times$ warped-down $k$ and we denote it as $m_{\text {gauge }}$. On the other hand, first KK mode of gauge fields with $(-+)$ boundary condition has slighter smaller mass than $m_{\text {gauge }}$.

KK fermion masses are determined by boundary condition and five-dimensional mass $m_{5}$, or $c=\frac{m_{5}}{k}$. For fermion fields with $c$ chosen such that the corresponding (would-be) zero mode is localized near the UV brane, we find that the KK mass is larger than KK gauge mass $m_{\text {gauge }}$, regardless of its boundary condition [assuming brane localized kinetic terms (BKT) are negligible]. This is the case for all leptonic fields, except for $\tilde{L}_{R}$. In order to produce SM neutrino mass, $\tilde{L}_{R}$ has $c \sim-0.3$ and resulting KK mass is naturally smaller than $m_{\text {gauge }}$. However, in the minimal setup, its mass is still bigger than $\frac{1}{2} m_{\text {gauge }}$, preventing the decay of $W_{R}^{(1)}$ into $N^{(1)}$ and $\tilde{\ell}^{(1)} \cdot{ }^{24}$ As is well known, turning on BKT's could lower the mass of corresponding KK modes [17]. In the Appendix, we show $O(1)$ BKT on the IR brane for $\tilde{L}_{R}$ can result in mass of $N^{(1)}$ and $\tilde{\ell}^{(1)}$ smaller than $\frac{1}{2} m_{\text {gauge }}$. Another interesting fact about BKT is that, both $N_{R}^{(1)}$ and $N_{L}^{(1)}$ can have similar coupling to gauge field $W_{R}^{(1)}$. In the absence of BKT, the coupling of $N_{L}^{(1)}$ to $W_{R}^{(1)}$ is mildly suppressed, i.e., by $O(1)$, as compared to $N_{R}^{(1)}$, since

\footnotetext{
${ }^{24}$ In this paper, superscript (1) and (0) are used to denote first KK mode and zero mode, respectively.
} 
$W_{R}$ is peaked near IR brane where $N_{L}^{(1)}$ has vanishing boundary condition. Combination of these two features opens new decay channels for KK $W_{R}^{(1)}$. Namely, the decay of $W_{R}^{(1)}$ into pair of $N_{R}^{(1)}$ and $\tilde{\ell}_{R}^{(1)}$ together with $N_{L}^{(1)}$ and $\tilde{\ell}_{L}^{(1)}$, which are our signal channels. For the sake of completeness, we mention that we do not introduce BKT for $\tilde{N}$ and hence on-shell decay of $\mathrm{KK} W_{R}^{(1)}$ into $\tilde{N}$ and its partner is forbidden.

A similar analysis can be applied to the quark sector: we find that, in the absence of BKT's, the only KK fermions which are a bit lighter than the $W_{R}^{(1)}$ (but still heavier than $1 / 2 m_{\text {gauge }}$ ) are the $S U(2)_{R}$ partners of $Q_{L}^{3}$ (like the case of $\tilde{L}_{R}$ above). We assume that BKT's for these states are not turned on (unlike for $\tilde{L}_{R}$ ) so that KK $W_{R}$ can not decay into pairs of these extra fermions. The decay channel for $W_{R}^{(1)}$ into $\mathrm{SM} Q_{L}^{3}$ and the above extra fermions is kinematically open; however, given the (roughly) flat profile of $Q_{L}^{3}$, this coupling is nonetheless suppressed compared to the coupling to $N^{(1)}$ and $\tilde{\ell}^{(1)}$ so that this decay mode can be neglected.

In this paper, we study the on-shell production of KK gauge bosons $W_{R}^{(1)}$ and its decays to $N^{(1)}-\tilde{\ell}^{(1)}$ pair. Particles heavier than $W_{R}^{(1)}$ are dropped for simplicity of study. Below, we summarize the spectrum of particles of interest:

$$
m_{\text {gauge }}>2 m_{\tilde{L}_{R}} \gg \text { mass of SM particles, }
$$

where KK gauge bosons included in our phenomenological study are $W_{L}^{(1)}, W_{R}^{(1)}$, and $Z^{(1)}, Z^{\prime(1)}$.

\section{TWO-SITE APPROACH TO NATURAL WARPED SEESAW}

The full five-dimensional warped model contains all the degrees of freedom with perturbative couplings. In this sense, it is fully calculable five-dimensional effective theory and any relevant questions can be answered by explicit computation. However, for a specific phenomenological search, only a finite subset of degrees of freedom and related couplings are involved and a simplified model consisted of only relevant particles and couplings will be much more efficient in practice. Two-site model of [10] provides one way to obtain a simplified four-dimensional effective theory by a consistent truncation of a full fivedimensional warped model to the first KK modes. This approach not only simplifies phenomenological studies, but also can encompass the phenomenology of a broader class of five-dimensional warped models, or its four-dimensional composite models, thereby allowing more inclusive/ systematic searches.

The two-site model, as the name suggests, consists of two sectors/sites: the elementary sector and the composite sector. The composite sector represents strong dynamics which confines at $O(\mathrm{TeV})$ scale, the scale where the scale invariance is spontaneously broken and composite resonances are "born." In principle, there will be towers of infinite resonances. However, as a phenomenological simplified model, only the lightest resonances, the relevant particles for the collider searches, are kept. Elementary sector, on the other hand, exhibits physics external to strong dynamics, but with couplings to the composite sector. These couplings induce mixing between elementary and composite states and upon diagonalization, this leads to massive mass eigenmodes, dual to first KK modes in five dimensions, and massless modes, dual to zero mode, i.e. SM fields. In this way, it is easily seen that both SM fields and the first KK modes of the five-dimensional model are generically the admixture of elementary and composite states, the amount of compositeness being determined by the size of the mixing at the $O(\mathrm{TeV})$ scale. Such a feature is known as "partial compositeness" in four-dimensional strong dynamics, a robust mechanism that solves flavour hierarchy problem of the SM. The five-dimensional dual of partial compositeness is the localization of the zero-mode profile along the extra dimension, localization near the IR (UV) brane being dual to more composite (elementary).

Two-site model of the natural warped seesaw that we reviewed in Sec. II can be described as follows. We begin by discussing the singlet neutrino $N_{R}$. In the elementary sector, there is elementary field $N_{R}$ that has large Majorana mass term $m_{N}$. In the composite sector, as already mentioned in the introduction, there is a composite singlet Dirac fermion $\left(\chi_{L}, \chi_{R}\right)$ with $O(\mathrm{TeV})$ Dirac mass. Finally, there is mass mixing between $N_{R}$ and $\chi_{L}$, i.e. they have the same quantum number, with the size of the mixing being characterized by the relevant scale, i.e. of the order of $O(\mathrm{TeV})$. These can be summarized by the following Lagrangian (dropping kinetic terms for simplicity):

$$
\begin{aligned}
\mathcal{L}_{\text {seesaw }} & =\mathcal{L}_{\text {elementary }}+\mathcal{L}_{\text {composite }}+\mathcal{L}_{\text {mixing }} \\
& =-\frac{m_{N}}{2} N_{R}^{T} C N_{R}-m_{D} \bar{\chi}_{L} \chi_{R}-\Delta \bar{\chi}_{L} N_{R}+\text { H.c. },
\end{aligned}
$$

where $m_{N}\left(m_{D}\right)$ is Majorana (Dirac) mass for elementary (composite) states and $\Delta$ is the mass mixing between the two. Both $m_{D}$ and $\Delta$ are $O(\mathrm{TeV})$, while $m_{N} \gg \mathrm{TeV}$. Largeness of the Majorana mass $m_{N}$ allows us to integrate out $N_{R}$, i.e. use equation of motion for $N_{R}$, to get

$$
\mathcal{L}_{\text {seesaw }}=-m_{D} \bar{\chi}_{L} \chi_{R}-\frac{m_{D} \Delta}{2 m_{N}} \chi_{L}^{T} C \chi_{L}+\text { H.c.. }
$$

Notice that integrating out $N_{R}$ generates the Majorana mass for left-handed $\chi_{L}$ of the composite singlet fermion, that is, it transmits lepton-number violation into the composite sector. Since $\frac{m_{D} \Delta}{m_{N}} \ll m_{D}$, it is clear that the composite fermion $\left(\chi_{L}, \chi_{R}\right)$ becomes pseudo-Dirac and the exchange of this pseudo-Dirac singlet fermion between the two lefthanded SM neutrinos then is the dynamical origin of the 
SM neutrino mass. Namely, it is the inverse seesaw for SM neutrino mass generation. Notice, however, that the way the small Majorana splitting is generated is by the "exchange" of super-heavy $N_{R}$, which can be viewed as a type I seesaw. As mentioned in Sec. II, since the Majorana splitting is much smaller than Dirac mass, we simply drop it and treat $\left(\chi_{L}, \chi_{R}\right)$ as a pure Dirac fermion for our collider analysis presented in Sec. V. For the rest of the study, we simply use $\left(N_{L}^{(1)}, N_{R}^{(1)}\right)$ to denote $\left(\chi_{L}, \chi_{R}\right)$ and put them and their $S U(2)_{\mathrm{R}}$ partner, denoted as $\left(\tilde{\ell}_{L}^{(1)}, \tilde{\ell}_{R}^{(1)}\right)$, in the doublet $\tilde{L}_{R}$.

For the rest of the model, following [10], we consider an elementary sector with elementary gauge group $\left[S U(2)_{\mathrm{L}} \times\right.$ $\left.U(1)_{\mathrm{Y}}\right]^{\text {elem }}$ and a composite sector with global symmetry $\left[S U(2)_{\mathrm{L}} \times S U(2)_{\mathrm{R}} \times U(1)_{\mathrm{X}}\right]^{\text {comp }}$. Focusing on the gauge sector first, there will be mixing terms between elementary gauge bosons and corresponding composite vector mesons, i.e. composite vector bosons associate with $\left[S U(2)_{\mathrm{L}} \times\right.$ $\left.U(1)_{\mathrm{Y}}\right]^{\text {comp }}$ subgroup of the full global symmetry of the composite sector. These mixing terms between elementary and composite vector bosons break both elementary and composite symmetries. However, it does so in a way that only one linear combination of the elementary gauge boson and composite vector meson gets a mass, leaving the other orthogonal combination being still massless. Namely, there is unbroken gauge symmetry which we identify as the SM gauge group $\left[S U(2)_{\mathrm{L}} \times U(1)_{\mathrm{Y}}\right]^{\mathrm{SM}}$. These massless (massive) mass eigenstates are dual to zero-(KK-)mode SM gauge boson arising in the five-dimensional model. In this way, we understand that there is mixing between elementary and composite vector bosons, allowing the coupling between elementary fermions and composite vector mesons.

On the other hand, since there exist no associated elementary gauge bosons, the charged vector mesons for $S U(2)_{\mathrm{R}}\left(W_{R}^{(1)}\right)$ and the one for $\left(U(1)_{\mathrm{R}} \times U(1)_{\mathrm{X}}\right) / U(1)_{\mathrm{Y}}$ $\left(Z^{\prime(1)}\right)$, i.e. orthogonal to $U(1)_{\mathrm{Y}}$, do not have mixing with elementary gauge bosons, i.e. purely composite. This feature is dual to the fact that the corresponding fivedimensional gauge bosons have odd boundary condition on the UV brane and have no zero mode. SM fermion fields are admixture of elementary and composite states (resulting from presence of mass terms along the lines of what was discussed for singlet neutrino above). In this study, just for simplicity, we treat all SM fermions to be purely elementary, except $\left(b_{L}, t_{L}\right)$ and $t_{R}$. As we discussed in Sec. II, the mass for KK modes of all SM fermions are higher than gauge KK; however, the KK modes from the $\tilde{L}_{R}$ multiplet in Eq. (16) are taken to be lighter. This is mapped into the two-site model by the fact that all "excited" composite modes of the SM fermions ${ }^{25}$ are heavier than composite vector mesons, thus for simplicity, we neglect them in what follows. However,

\footnotetext{
${ }^{25}$ Note that this also applies to the composites with which the external right-handed charged lepton mixes, i.e., corresponding to the field $\ell$ from the $L_{R}$ multiplet in Eq. (16).
}

the composite $S U(2)_{\mathrm{R}}$ doublet containing the singlet neutrino (discussed above) is light. Higgs is chosen to be pure composite state as a standard choice. The diagonalized Lagrangian before EWSB (see next section for this effect) containing all these degrees of freedom is given by

$$
\mathcal{L}=\mathcal{L}_{\text {gauge }}+\mathcal{L}_{\text {fermion }}+\mathcal{L}_{\text {Higgs }} .
$$

where we provide each part below one by one. First of all, $\mathcal{L}_{\text {gauge }}$ is given by

$$
\begin{aligned}
\mathcal{L}_{\text {gauge }}= & -\frac{1}{4} F_{\mu \nu}^{2}+\frac{1}{2}\left(D_{\mu} \rho_{\nu} D_{\nu} \rho_{\mu}-D_{\mu} \rho_{\nu} D_{\mu} \rho_{\nu}\right)+\frac{m_{\star}^{2}}{2} \tilde{\rho}_{\mu}^{2} \\
& +\frac{m_{\star}^{2}}{2 \cos ^{2} \phi} \rho_{\mu}^{2}+\frac{i g}{2} F_{\mu \nu}\left[\rho_{\mu}, \rho_{\nu}\right],
\end{aligned}
$$

where $\rho_{\mu}=\left(W_{L \mu}^{(1)}, B_{\mu}^{(1)}\right)$ (using the five-dimensional notation, i.e., KK of SM gauge fields), $\tilde{\rho}_{\mu}=\left(W_{R \mu}^{(1)}, Z_{\mu}^{\prime(1)}\right)$ (nonSM gauge bosons) and $A_{\mu}=\left(W_{L \mu}^{(0)}, B_{\mu}^{(0)}\right)$ (the SM gauge bosons). $F_{\mu \nu}$ is the field strength of $A_{\mu}$. All covariant derivatives in the Lagrangians are with respect to the unbroken SM gauge group, namely $D_{\mu}=\partial_{\mu}-i g A_{\mu}$. All the vector fields and field strength in the Lagrangians in this section are in matrix forms. Final results can be obtained by taking traces of Lagrangians with appropriate normalization. Gauge couplings are SM gauge couplings $g=\left(g_{W}, g_{Y}\right)$ and composite gauge couplings $g_{\star}=\left(g_{\star W}, g_{\star Y}\right)$, and $\tilde{g}_{\star}=\left(g_{\star R}, g_{\star Z^{\prime}}\right)$, where $g_{\star Y}=\frac{g_{\star R} g_{\star X}}{\sqrt{g_{\star R}^{2}+g_{\star X}^{2}}}$. The elementarycomposite mixing angle $\phi=\left(\phi_{W}, \phi_{Y}\right)$ is defined as $\sin \phi=\frac{g}{g_{\star}}$. Finally, $m_{\star}$ denotes the composite spin-1 mass before mixing with elementary states, hence this is also the mass for $\tilde{\rho}$ 's (i.e., $W_{R}^{ \pm}$and $Z^{\prime}$ ) who do not have such mixing. Whereas, for composite partners of $S M$ gauge bosons, i.e., $\rho$ 's, the mass is modified by this mixing as indicated above.

Note that we are providing phenomenologically most relevant terms only, dropping terms with three or more $\rho$ 's or $\tilde{\rho}$ 's. This is valid approximation since we are working to leading order and, at the leading order, only two body decays of the heavy particles, e.g. $\rho$ 's or $\tilde{\rho}$ 's, are relevant.

Moving onto the fermion sector, $\mathcal{L}_{\text {fermion }}$ (for the fields relevant for our collider study) is given by

$$
\begin{aligned}
\mathcal{L}_{\text {fermion }}= & \bar{\psi}_{\mathrm{SM}} i \not \psi_{\mathrm{SM}}+\overline{\tilde{L}}_{R}\left(i \not D-m_{D}\right) \tilde{L}_{R} \\
& -g \tan \phi \bar{\psi}_{\text {light }} \rho_{\mu} \gamma^{\mu} \psi_{\text {light }} \\
& +g\left(\sin ^{2} \phi_{Q_{L}^{3}} \cot \phi-\cos ^{2} \phi_{Q_{L}^{3}} \tan \phi\right) \bar{Q}_{L}^{3} \rho_{\mu} \gamma^{\mu} Q_{L}^{3} \\
& +g_{\star Y} \bar{t}_{R} B_{\mu}^{(1)} \gamma^{\mu} t_{R}+\tilde{g}_{\star} \sin ^{2} \phi_{Q_{L}^{3}}\left(\bar{b}_{L} Z_{\mu}^{\prime} \gamma^{\mu} b_{L}+\bar{t}_{L} Z_{\mu}^{\prime} \gamma^{\mu} t_{L}\right) \\
& +g_{\star Z^{\prime}} \bar{t}_{R} Z_{\mu}^{\prime} \gamma^{\mu} t_{R}+\tilde{g}_{\star} \overline{\tilde{L}}_{R} \tilde{\rho}_{\mu} \gamma^{\mu} \tilde{L}_{R}+g_{\star} \overline{\tilde{e}} B_{\mu}^{(1)} \gamma^{\mu} \tilde{e}
\end{aligned}
$$

where $\psi_{\mathrm{SM}}$ denotes all SM fermions and $\psi_{\text {light }}=\psi_{\mathrm{SM}^{-}}$ $\left\{Q_{L}^{3}, t_{R}\right\}$, i.e. light $\mathrm{SM}$ fermions. It is understood that all 
couplings should be multiplied by appropriate charges to get final coupling, which we do not show explicitly. The mixing angle between elementary and composite states of the associated fermion $\psi$ is denoted by $\phi_{\psi}$, with $\cos \phi_{\psi}=$ $1(0)$ corresponds to pure elementary (composite). The specific representations of fermions are discussed in Sec. II. As mentioned earlier, here we assume that light SM fermions are purely elementary, i.e. $\cos \phi_{\psi_{\text {light }}}=1$ (corresponding to the zero modes being localized near the UV brane in the five-dimensional model), and $Q_{L}^{3}$ is slightly composite (roughly flat profile) and $t_{R}$ is fully composite (localized near the IR brane), i.e. $\cos \phi_{t_{R}}=0$.

Finally, $\mathcal{L}_{\text {Higgs }}$ has the form

$$
\mathcal{L}_{\text {Higgs }}=\left|D_{\mu} \boldsymbol{\Phi}+i g \cot \phi \rho_{\mu} \boldsymbol{\Phi}-i \tilde{g}_{\star} \boldsymbol{\Phi} \tilde{\rho}_{\mu}\right|^{2}-V(\boldsymbol{\Phi})-y \bar{L} \boldsymbol{\Phi} \tilde{L}_{R},
$$

where $\boldsymbol{\Phi}$ denotes the Higgs bidoublet $\boldsymbol{\Phi}=\left(i \sigma_{2} \Phi^{*}, \Phi\right)$. $V(\boldsymbol{\Phi})$ is the Higgs potential. $L$ is the SM lepton doublet, and $y$ is the Yukawa coupling constant.

Note the sizable couplings of light quarks to $\rho$ 's, i.e., excited SM gauge bosons, in the second line of Eq. (23); these are nonetheless suppressed compared to the SM gauge couplings by the smallness of the elementary-composite mixing factor and correspond to the profile of the gauge KK modes at the UV brane in the five-dimensional picture. In any case, it is these couplings that will be relevant for production of the spin-1 states at the LHC. On the other hand, the coupling of light quarks to non-SM gauge bosons, i.e., $W_{R}^{ \pm(1)}$ and $Z^{\prime(1)}$ (denoted collectively by $\tilde{\rho}$ ) is negligible, due to the absence of the elementary counterparts (and dual to profile of those gauge KK vanishing on the UV brane). However, as we will see below, a sizable coupling of light quarks to $W_{R}^{ \pm(1)}$ and $Z^{\prime(1)}$ will be generated due to EWSB effects. As far as decay of spin-1 states is concerned, it is the couplings in last line of Eq. (23), i.e., to composite leptons, to top quark in line above it and to Higgs particles from Eq. (24) which dominate.

\section{A. Higgs-induced gauge mixing}

When Higgs gets a VEV and the electroweak symmetry is spontaneously broken, it generates mixing among gauge bosons and fermions. In order to obtain mass spectrum, then, mass matrices should be diagonalized. In this section, we shall discuss the diagonalization of mass matrices and show, in particular, that the mass eigenstates of massive vector bosons consist of $O(1)$ components of both $W_{L}^{(1)}$ and $W_{R}^{(1)}$. That is, EWSB induces a significant mixing between $W_{L}^{(1)}$ and $W_{R}^{(1)}$, and this will be the main production channel for $W_{R}^{(1)}$ (as mentioned earlier). We choose $g_{\star W}$ and $g_{\star R}$ to be the same for our benchmark points.

The mass matrix for charged vector bosons is given by

$$
\left(W_{L}^{+(0)} W_{L}^{+(1)} W_{R}^{+(1)}\right) \mathcal{M}^{2}\left(W_{L}^{-(0)} W_{L}^{-(1)} W_{R}^{-(1)}\right)^{T} \text {, }
$$

where

$$
\mathcal{M}^{2}=\frac{1}{4}\left(\begin{array}{ccc}
g_{W}^{2} v^{2} & g_{W}^{2} v^{2} \cot \phi_{W} & -g_{W} g_{\star W} v^{2} \\
g_{W}^{2} v^{2} \cot \phi_{W} & 4 \frac{m_{\star}^{2}}{\cos ^{2} \phi_{W}}+\left(g_{W} \cot \phi_{W} v\right)^{2} & -g_{W} \cot \phi_{W} g_{\star W} v^{2} \\
-g_{W} g_{\star W} v^{2} & -g_{W} \cot \phi_{W} g_{\star W} v^{2} & 4 m_{\star}^{2}+\left(g_{\star W} v\right)^{2}
\end{array}\right) .
$$

Note that we assume the same purely composite mass $m_{\star}$ for all composite gauge fields, i.e., before mixing with elementary states; this mixing does perturb the mass for excited $S M$ gauge bosons as seen above. We will return to the more general case of nondegenerate composites in a follow-up paper [11].

Performing explicit diagonalization of the above matrix analytically can be quite challenging. However, we can use the following method to get an approximate result. Our procedure will be valid as the following relations hold:

$$
\frac{1}{4} g_{\star}^{2} v^{2} \ll m_{\star}^{2} \quad \text { and } \quad g \ll g_{\star}, \quad \tilde{g}_{\star} .
$$

We demand that the mass matrix can be fully diagonalized by the following transformation by $U$,

$$
U^{\dagger} \mathcal{M}^{2} U=\mathcal{M}_{\text {diag }}^{2},
$$

where

$$
U=U_{12} U_{13} U_{23}
$$

with

$$
\begin{aligned}
U_{12} & =\left(\begin{array}{ccc}
c & s & 0 \\
-s & c & 0 \\
0 & 0 & 1
\end{array}\right) \\
U_{13} & =\left(\begin{array}{ccc}
C & 0 & S \\
0 & 1 & 0 \\
-S & 0 & C
\end{array}\right) \\
U_{23} & =\left(\begin{array}{ccc}
1 & 0 & 0 \\
0 & c_{\star} & s_{\star} \\
0 & -s_{\star} & c_{\star}
\end{array}\right) .
\end{aligned}
$$




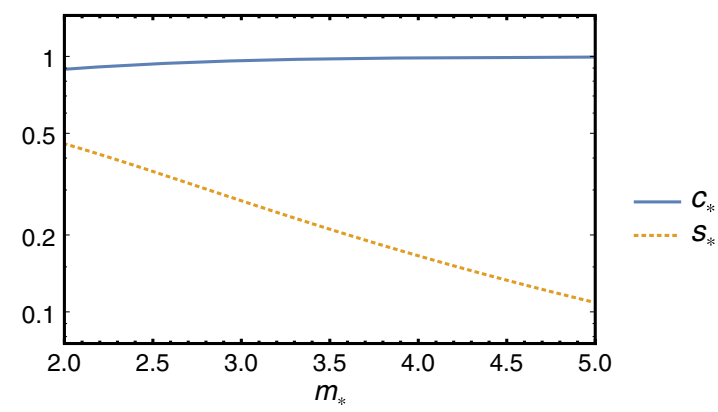

FIG. 2. The figure denotes sine and cosine of mixing $\operatorname{angle}\left(s_{\star}, c_{\star}\right)$ between $W_{L}^{(1)}$ and $W_{R}^{(1)}$ as a function of $m_{\star}$, with $g_{\star W}=g_{\star R}=3$. SM parameters are chosen to be standard values $g_{W}=0.65$ and $v=246 \mathrm{GeV}$.

Here $s, S, s_{\star}$ represent the sines of $\theta_{12}, \theta_{13}$, and $\theta_{23}$, whereas $c, C, c_{\star}$ represent cosines of associated angles. Making use of the approximations of Eq. (27), one readily finds that

$$
\begin{aligned}
\tan 2 \theta_{12} & \approx \frac{2 g_{W} g_{\star W} v^{2}}{4 m_{\star}^{2}+g_{\star W}^{2} v^{2}} \\
\tan 2 \theta_{13} & \approx \frac{-2 g_{W} g_{\star W} v^{2}}{4 m_{\star}^{2}+g_{\star W}^{2} v^{2}} \\
\tan 2 \theta_{23} & \approx \frac{2 g_{\star W}^{2} v^{2}}{4 \sin ^{2} \phi_{W} m_{\star}^{2}} .
\end{aligned}
$$

The last formula corresponds to the mixing between two composite vector bosons, $W_{L}^{(1)}$ and $W_{R}^{(1)}$. Since $\frac{1}{4} g_{\star}^{2} v^{2} \ll m_{\star}^{2}$, we would naively think that this mixing is small. However, using $\sin ^{2} \phi_{W}=\frac{g_{W}^{2}}{g_{* W}^{2}}$, we get

$$
\tan 2 \theta_{23} \approx \frac{g_{\star W}^{2} v^{2}}{2\left(\frac{g_{W}}{g_{\star}} m_{\star}\right)^{2}},
$$

and, as far as $g \ll g_{\star}$, this mixing angle is $O(1)$ ! That is, in most of the parameter space of interest, we get a significant mixing between $W_{L}^{(1)}$ and $W_{R}^{(1)}$ (see Fig. 2). The same feature was pointed out in [13] using the full five-dimensional model, instead of the two-site model presented here. The origin of the above large mixing can be understood as follows. From the mass matrix Eq. (26), one can find

$$
m_{W_{L}^{(1)}}^{2}-m_{W_{R}^{(1)}}^{2}=\tan ^{2} \phi_{W} m_{\star}^{2},
$$

and using $\sin ^{2} \phi_{W} \approx \tan ^{2} \phi_{W} \approx \frac{g_{W}^{2}}{g_{\star W}^{2}} \ll 1$, Eq. (32) can be rewritten as follows.

$$
\tan 2 \theta_{23} \approx \frac{\frac{1}{2} g_{\star W}^{2} v^{2}}{\left(M_{W_{L}^{(1)}}^{2}-M_{W_{R}^{(1)}}^{2}\right)} .
$$

This expression manifests the fact that the large mixing arises when $W_{L}^{(1)}$ and $W_{R}^{(1)}$ have almost degenerate masses, i.e. the mass gap $m_{W_{L}^{(1)}}^{2}-m_{W_{R}^{(1)}}^{2}$ is suppressed or comparable to $g_{\star W}^{2} v^{2}$.

The relation with the mass basis denoted by $W, W_{L}$, and $W_{R}$ is given by

$$
\left(\begin{array}{c}
W \\
W_{L} \\
W_{R}
\end{array}\right)=U_{23}^{\dagger} U_{13}^{\dagger} U_{12}^{\dagger}\left(\begin{array}{c}
W_{L}^{(0)} \\
W_{L}^{(1)} \\
W_{R}^{(1)}
\end{array}\right) .
$$

Since $\frac{1}{4} g_{\star}^{2} v^{2} \ll m_{\star}^{2}, \theta_{12} \approx \theta_{13} \ll 1$, we can approximate $s=S$. After dropping all terms with two or more $s$ or $S$, we can get

$$
\begin{gathered}
W=C\left(c W_{L}^{(0)}-s W_{L}^{(1)}\right)-S W_{R}^{(1)} \\
W_{L} \approx s W_{L}^{(0)}+c\left(c_{\star} W_{L}^{(1)}-s_{\star} W_{R}^{(1)}\right) \\
W_{R} \approx S W_{L}^{(0)}+C\left(s_{\star} W_{L}^{(1)}+c_{\star} W_{R}^{(1)}\right) .
\end{gathered}
$$

The typical size of these mixing angles is

$$
\begin{aligned}
s \approx|S| & \sim \frac{g_{W} g_{\star W} v^{2}}{4 m_{\star}^{2}} \ll 1 \\
s_{\star} & \sim \frac{g_{\star W}^{2} v^{2}}{4\left(\frac{g_{W}}{g_{\star}} m_{\star}\right)^{2}} \sim 1 .
\end{aligned}
$$

Given the above large mixing between $W_{L, R}^{(1)}$ induced by the Higgs VEV, it is clear that light quarks will now couple similarly (and significantly) to both the mass eigenstates, cf. in the basis prior to EWSB, the coupling to one of the states, i.e., $W_{R}^{(1)}$, was negligible.

The masses of the physical states will also be perturbed due to the EWSB effects. Here, for simplicity, we kept only two massive states $W_{L}^{(1)}$ and $W_{R}^{(1)}$ to obtain the mass splitting, assuming that the small fraction of $W^{(0)}$ in the mass eigenstate does not make any difference. With such mass splitting, the physical mass for $W_{L}$ and $W_{R}$ is given by

$m_{W_{L / R}}^{2} \approx m_{\star}^{2}+\frac{1}{4} g_{\star W}^{2} v^{2} \pm \sqrt{\frac{g_{W}^{4}}{4 g_{\star W}^{4}} m_{\star}^{4}+\frac{1}{16} g_{\star W}^{4} v^{4}}$,

where the $+(-)$ sign is for $m_{W_{L}}\left(m_{W_{R}}\right)$.

Similar analysis can be done for neutral gauge bosons. The mass matrix is given by

$$
\left(Z^{(0)} Z^{(1)} Z^{\prime(1)}\right) \frac{1}{2} \mathcal{M}^{2}\left(Z^{(0)} Z^{(1)} Z^{\prime(1)}\right)^{T},
$$

where 


$$
\mathcal{M}^{2}=\frac{1}{4}\left(\begin{array}{ccc}
g_{Z}^{2} v^{2} & g_{Z}^{2} v^{2} \cot \phi_{Z} & -g_{Z} g_{\star Z^{\prime}} c^{\prime 2} v^{2} \\
g_{Z}^{2} v^{2} \cot \phi_{Z} & 4 \frac{m_{\star}^{2}}{\cos ^{2} \phi_{Z}}+\left(g_{Z} \cot \phi_{Z} v\right)^{2} & -g_{Z} \cot \phi_{Z} g_{\star Z^{\prime}} c^{\prime 2} v^{2} \\
-g_{Z} g_{\star Z^{\prime}} c^{\prime 2} v^{2} & -g_{Z} \cot \phi_{Z} g_{\star Z^{\prime}} c^{\prime 2} v^{2} & 4 m_{\star}^{2}+\left(g_{\star Z^{\prime}} c^{\prime 2} v\right)^{2}
\end{array}\right)
$$

Here $c^{\prime}=\sqrt{1-\tan ^{2} \theta_{W}}$ and $\theta_{W}$ is Weinberg angle in the composite sector. We assume that the composite sector has the same Weinberg angle as the SM. Mass eigenstates are denoted by $Z, Z_{1}$, and $Z^{\prime}$, and are related to gauge basis fields by

$$
\begin{aligned}
Z & =C\left(c Z^{(0)}-s Z^{(1)}\right)-S Z^{\prime(1)} \\
Z_{1} & \approx s Z^{(0)}+c\left(c_{\star} Z^{(1)}-s_{\star} Z^{\prime(1)}\right) \\
Z^{\prime} & \approx S Z^{(0)}+C\left(s_{\star} Z^{(1)}+c_{\star} Z^{\prime(1)}\right) .
\end{aligned}
$$

The typical size of the mixing angles is

$$
\begin{gathered}
s \sim \frac{g_{Z} g_{\star Z} v^{2}}{4 m_{\star}^{2}} \ll 1 \\
|S| \sim \frac{g_{Z} g_{\star Z^{\prime}} c^{\prime 2} v^{2}}{4 m_{\star}^{2}} \ll 1 \\
s_{\star} \sim \frac{g_{\star Z} g_{\star Z^{\prime}} c^{\prime 2} v^{2}}{4\left(\frac{g_{Z}}{g_{\star Z}} m_{\star}\right)^{2}} \sim 1 .
\end{gathered}
$$

Assuming $g_{\star Z} \approx g_{\star Z^{\prime}} c^{\prime 2}$, the spectrum of the mass eigenstate is

$$
m_{Z_{1} / Z^{\prime}}^{2} \approx m_{\star}^{2}+\frac{1}{4} g_{\star Z}^{2} v^{2} \pm \sqrt{\frac{g_{Z}^{4}}{4 g_{\star Z}^{4}} m_{\star}^{4}+\frac{1}{16} g_{\star Z}^{4} v^{4}},
$$

where the $+(-)$ sign is for $m_{Z_{1}}\left(m_{Z^{\prime}}\right)$.

\section{B. Lepton mixing}

Apart from mixing in the gauge sector, EWSB also induces mixing in the fermion sector. As discussed earlier, composite "excited" modes for SM particles are neglected because they are heavier than composite vector bosons and composite states of singlet neutrino. For this reason, we focus on the mixing among SM lepton doublet $L$ and the composite $S U(2)_{R}$ doublet $\tilde{L}_{R}$. For simplicity, here we assume flavor diagonal Yukawa coupling and mass matrix for singlet neutrinos. In the next section, we will discuss related flavour issues.

The relevant parts of the Lagrangian containing Yukawa coupling of $L$ and $\tilde{L}_{R}$ are as follows:

$$
-\mathcal{L} \ni y \bar{L}_{i} \boldsymbol{\Phi} \tilde{L}_{R i}+m_{D} \overline{\tilde{L}}_{R i} \tilde{L}_{R i}
$$

where $y$ is the Yukawa coupling and $i$ denotes the generation index of leptons, $i=\{e, \mu, \tau\} . m_{D}$ is the Dirac mass for composite $\tilde{L}_{i}$. The elementary (composite) $S U(2)_{\mathrm{L}}\left(S U(2)_{\mathrm{R}}\right)$ doublet $L\left(\tilde{L}_{R}\right)$ is defined as

$$
\begin{aligned}
L & =\left(\nu_{L}^{(0)}, \ell_{L}^{(0)}\right) \\
\tilde{L}_{R} & =\left(N^{(1)}, \tilde{\ell}^{(1)}\right) .
\end{aligned}
$$

When the Higgs field gets a VEV, the Lagrangian Eq. (44) generates neutrino mixing as can be seen from

$\frac{y v}{\sqrt{2}} \bar{\nu}_{L}^{(0)} N_{R}^{(1)}+m_{D} \bar{N}_{L}^{(1)} N_{R}^{(1)}=m_{D}\left(\bar{N}_{L}^{(1)}+\frac{y v}{\sqrt{2} m_{D}} \bar{\nu}_{L}^{(0)}\right) N_{R}^{(1)}$.

From this, we can obtain physical mass eigenstates denoted as $N_{L}, N_{R}$, and $\nu_{L}$ :

$$
\begin{aligned}
& N_{L} \approx N_{L}^{(1)}+V_{\ell N} \nu_{L}^{(0)} \\
& N_{R}=N_{R}^{(1)} \\
& \nu_{L} \approx \nu_{L}^{(0)}-V_{\ell N} N_{L}^{(1)},
\end{aligned}
$$

where the mixing is given by $V_{\ell N}=\frac{y v}{\sqrt{2} m_{D}}$. The same Lagrangian also introduces charged lepton mixing after EWSB (we can safely neglect the SM charged lepton Yukawa coupling or mass term here as compared to the others):

$\frac{y v}{\sqrt{2}} \bar{\ell}_{L}^{(0)} \tilde{\ell}_{R}+m_{D} \overline{\tilde{\ell}}_{L}^{(1)} \tilde{\ell}_{R}^{(1)}=m_{D}\left(\overline{\tilde{\ell}}_{L}^{(1)}+\frac{y v}{\sqrt{2} m_{D}} \bar{\ell}_{L}^{(0)}\right) \tilde{\ell}_{R}^{(1)}$.

Again, from this, we can obtain physical mass eigenstates denoted as $\tilde{\ell}_{L}, \tilde{\ell}_{R}$, and $\ell_{L}$ :

$$
\begin{aligned}
& \tilde{\ell}_{L} \approx \tilde{\ell}_{L}^{(1)}+V_{\ell N} \ell_{L}^{(0)} \\
& \tilde{\ell}_{R}=\tilde{\ell}_{R}^{(1)} \\
& \ell_{L} \approx \ell_{L}^{(0)}-V_{\ell N} \tilde{\ell}_{L}^{(1)} .
\end{aligned}
$$

In principle, there is a similar effect from mixing of SM $S U(2)_{L}$ singlet charged leptons (after EWSB) with 
composite $S U(2)_{L}$ doublets; however, since we assumed that such composites are heavy, we can neglect it. Moreover, electrons and neutrinos have the same mixing $V_{\ell N}$. This is because (1) $N$ and $\tilde{\ell}$ are in the same $S U(2)_{R}$ doublet with the same mass $m_{D}$, together $\nu_{L}$ and $l_{L}$ being in the same $S U(2)_{L}$ doublet and (2) these two mixings originate from the same Yukawa coupling.

\section{OVERVIEW OF LHC SIGNALS}

In this section, based on our discussion in previous section, we first summarize couplings relevant to our collider study in Sec. V. Then, we specify the choice of parameters used for actual analysis, together with related bounds. We then discuss production and dominant decay channels of heavy gauge bosons, i.e. $W_{L}$ and $W_{R}$. In particular, we show that $W_{R} \rightarrow N \tilde{\ell}$ is indeed the dominant decay channel for most of the parameter space of interest, providing abundance production of $N$ and $\tilde{\ell}$. We end our discuss by providing formulas for decay widths of $N$ and $\tilde{\ell}$.

\section{A. Relevant couplings}

There are three types of couplings that we need to consider: (1) couplings between $W_{L} / W_{R}$ and SM fermions (2) couplings of $W_{L} / W_{R}$ to $N-\tilde{\ell}$ pair, and (3) couplings among $N(\tilde{\ell})$ —SM $H$, longitudinal $W / Z$-SM lepton $\ell(\nu)$ via Yukawa coupling.

(1) The first type of coupling can be obtained by using Eq. (23) and EWSB-induced mixing Eq. (36):

$$
\begin{aligned}
\delta \mathcal{L}_{(1)}= & -\frac{1}{\sqrt{2}} \frac{g_{W}^{2}}{g_{\star}}\left(c_{\star} W_{L \mu}^{+}-s_{\star} W_{R \mu}^{+}\right) \bar{\psi}_{L} \gamma^{\mu} \psi_{L}^{\prime} \\
& +\frac{1}{\sqrt{2}}\left(\sin ^{2} \phi_{Q_{L}^{3}} g_{\star W}-\cos ^{2} \phi_{Q_{L}^{3}} \frac{g_{W}^{2}}{g_{\star W}}\right) \\
& \times\left(c_{\star} W_{L \mu}^{+}-s_{\star} W_{R \mu}^{+}\right) \bar{t}_{L} \gamma^{\mu} b_{L}+\text { H.c. },
\end{aligned}
$$

where $\psi_{L}\left(\psi_{L}^{\prime}\right)$ denotes left chirality of light SM fermions. These couplings are responsible for the production of $W_{L}$ and $W_{R}$ via light quarks fusion inside proton. Notice that they suppressed by the factor $\frac{g_{W}}{g_{* W}}$ and mixing angle compared to fourdimensional LR models. However, as we will show in Sec. V, these couplings, even with such suppressions, still render large enough signal production to be discoverable in near future.

(2) The second type of coupling can be understood from Eq. (23) and mixing induced by EWSB Eq. (36):

$$
\delta \mathcal{L}_{(2)}=\frac{g_{\star W}}{\sqrt{2}} s_{\star} W_{L \mu}^{+} \bar{N} \gamma^{\mu} \tilde{\ell}+\frac{g_{\star W}}{\sqrt{2}} c_{\star} W_{R \mu}^{+} \bar{N} \gamma^{\mu} \tilde{\ell}+\text { H.c.. }
$$

These couplings lead to the decays of $W_{L}$ and $W_{R}$ to $N$ and $\tilde{\ell}$.

(3) The third type of couplings are similarly obtained from Eqs. (23), (24) and mixing induced by EWSB Eqs. (47), (49):

$$
\begin{aligned}
\delta \mathcal{L}_{(3)}= & \frac{g_{W}}{\sqrt{2}} V_{\ell N} W_{\mu}^{+} \bar{N}_{L} \gamma^{\mu} \ell_{L}+\{N \leftrightarrow \nu ; \ell \leftrightarrow \tilde{\ell}\} \\
& +\frac{g_{Z}}{2} V_{\ell N} Z_{\mu} \bar{N}_{L} \gamma^{\mu} \nu_{L}-\frac{y}{\sqrt{2}} H \bar{N}_{R} \nu_{L} \\
& +\{N \leftrightarrow \tilde{\ell} ; \nu \leftrightarrow \ell\}+\text { H.c.. }
\end{aligned}
$$

These couplings lead to the decays of $N$ and $\tilde{\ell}$ to physical Higgs $(H)$ and $W / Z$ and $\ell / \nu$.

\section{B. Parameter choice}

The composite sector generally contains many parameters, such as $g_{\star}$ 's and $\tilde{g}_{\star}$ 's. In our study, as our benchmark points, we assume all $\phi$ 's are the same, i.e. the ratio $g / g_{\star}$ are the same for all SM gauge groups. This choice is mainly for the sake of simplicity, and other choices with small variations will not lead to much difference in the final results. Besides, we fix $g_{\star W}=g_{\star R}$, or equivalently we assume there exists $Z_{2}$ symmetry connecting $S U(2)_{\mathrm{L}}$ and $S U(2)_{\mathrm{R}}$. This is well motivated by the consistency with EW precision tests, e.g. to suppress the corrections to the coupling $Z \rightarrow b \bar{b}$. With these choices, we are left with basically only one free gauge coupling in composite sector $g_{\star W}$. The composite gauge coupling $g_{\star W}$ has a lower bound $\sim 3$, which comes from the requirement that the Landau pole does not appear below the GUT scale. We choose $g_{\star W}=3$ as a benchmark points.

The mass parameter $m_{\star}$ for heavy gauge bosons is constrained by EW precision tests. With extended symmetry group $S U(2)_{\mathrm{L}} \times S U(2)_{\mathrm{R}} \times U(1)_{\mathrm{X}}$, the bound is given by $\gtrsim 3 \mathrm{TeV}$ in most parts of parameter space. Partly motivated by the discoverability at the LHC, we choose $m_{\star}=2 \mathrm{TeV}$ for our study. Such a low mass might be achieved in some corners of the parameter space or by invoking additional effects in EW precision tests (see for example [18]). Also, we choose $\sin ^{2} \phi_{Q_{L}^{3}}=0.21$, which may be on the edge of constraints from the EW precision test. This, again, can potentially be allowed by introducing additional structure in the model.

Moreover, the mass of composite gauge bosons is tightly constrained by diboson searches at ATLAS [19] and CMS [20]. In our model, $W_{L}^{(1)}$ will dominantly decay to diboson, just like the standard composite models. However, the branching ratio of $W_{R}^{(1)}$ to diboson is diluted by the new decay channel to $N$ and $\tilde{\ell}$. After EWSB, these two modes mix to render the mass eigenstates $W_{L} / W_{R}$. Assuming $W_{L}^{(1)}$ constitutes the dominant component of the mass eigenstate $W_{L}$, it can be understood that the dominant decay channels for $W_{L}$ will still be diboson. We explicitly 
checked that, in our benchmark points, $m_{\star}=2 \mathrm{TeV}$ is still consistent with current diboson searches.

Next, $\left|V_{\ell N}\right|^{2}$ is constrained by various experiments and the results are summarized in [21]. As we mentioned above, we assume flavor diagonal Yukawa coupling and mass matrix for singlet neutrinos. Naively, then, this model will not be able to accommodate the observed SM neutrino mixing and in this sense it is to be considered as just a toy model. Even with this assumption, there will be shift in coupling of SM neutrinos to the $Z$ boson, which is constrained by EW precision tests as $\left|V_{\ell N}\right|^{2} \lesssim 10^{-3}$ and we must take this into account. Realistically, i.e. considering nonvanishing off-diagonal entries for Yukawa and in singlet neutrino mass matrix, there will be SM neutrino mixing (as needed) and contributions to other lepton flavor-violating observables such as $\mu \rightarrow e \gamma$. In this generic case, $\left|V_{\ell N}\right|^{2}$ is constrained to be as strong as $10^{-5}$ (see, for example, the third reference in [21]). However, we can invoke some sort of flavor symmetry (see, for example, first reference in [3]) in order to suppress the latter (unwanted) effects; in particular, when the singlets are degenerate, there is a cancellation in $\mu \rightarrow e \gamma$. In this case, the constraint from lepton flavor-violation on the size of $\left|V_{\ell N}\right|^{2}$ is relaxed, perhaps allowing values as large as the (irreducible) EW precision tests bound of $10^{-3}$. Having this in mind, we choose the $\left|V_{\ell N}\right|^{2}=10^{-3}$ for all three generations. We would like to emphasize that the general features and rate of the signal in the actual/realistic model will not be much different from the case of our simplified assumption here.

In order for $W_{L}$ and $W_{R}$ to be able to decay to the pair $N-\tilde{\ell}, m_{\tilde{L}_{R}}$ needs to be smaller than half of $m_{\star}$. In principle, this mass is also constrained correlated with constraints of $\left|V_{\ell N}\right|^{2}$. With the choice we make $\left|V_{\ell N}\right|^{2}=10^{-3}$, however, there is no effective bound on $m_{\tilde{L}_{R}}$. Nevertheless, given that heavy gauge bosons, $N$, and $\tilde{\ell}$ all "live" in the same composite sector, too big hierarchy between $m_{\tilde{L}_{R}}$ and $m_{\star}$ will lead to unwanted tuning. Taking into account all these considerations, we choose $m_{\tilde{L}_{R}}=750 \mathrm{GeV}$ in our study.

\section{C. $W_{L} / W_{R}$ production and decay}

As mentioned already, $W_{L}$ and $W_{R}$ are produced via couplings in Eq. (50). Decay width for dominant decay channels are shown below, which are computed using couplings Eq. (51). Since the analytic expression for decay widths of mass eigenstates $W_{L}$ and $W_{R}$ are quite complicated, we instead provide expressions for gauge fields in gauge basis, namely $W_{L}^{(1)}$ and $W_{R}^{(1)}$. This will be sufficient for the purpose of our discussion. All the decay widths present in this paper are given with the assumption $m_{\star}>2 m_{\tilde{L}_{R}} \gg$ mass of SM particles, thus masses of SM particles are reasonably neglected. Decay widths for $W_{L}^{(1)}$ are given by

$$
\begin{aligned}
\Gamma\left(W_{L}^{(1)} \rightarrow W H / W Z\right) & \approx g_{\star W}^{2} \frac{m_{\star}}{192 \pi \cos \phi_{W}} \\
\Gamma\left(W_{L}^{(1)} \rightarrow t b\right) & \approx g_{\star W}^{2} \sin ^{4} \phi_{Q_{L}^{3}} \frac{m_{\star}}{16 \pi \cos \phi_{W}} \\
\Gamma\left(W_{L}^{(1)} \rightarrow \psi \psi^{\prime}\right) & \approx N_{c} \frac{g_{W}^{4}}{g_{\star W}^{2}} \frac{m_{\star}}{48 \pi \cos \phi_{W}},
\end{aligned}
$$

where $\psi$ and $\psi^{\prime}$ denote SM fermions, and $N_{c}$ shows the degree of freedom of corresponding fermion $\psi$ : 3 for quarks and 1 for leptons. Next, decay widths for $W_{R}^{(1)}$ are given by

$$
\begin{aligned}
\Gamma\left(W_{R}^{(1)} \rightarrow N_{i} \tilde{\ell}_{i}\right) & \approx g_{\star W}^{2}\left(1+2 \frac{m_{\tilde{L}_{R}}^{2}}{m_{\star}^{2}}\right) \sqrt{1-4 \frac{m_{\tilde{L}_{R}}^{2}}{m_{\star}^{2}}} \frac{m_{\star}}{24 \pi} \\
\Gamma\left(W_{R}^{(1)} \rightarrow W Z / W H\right) & \approx g_{\star W}^{2} \frac{m_{\star}}{192 \pi},
\end{aligned}
$$

where subscript $i$ is generation index.

From Eqs. (53) and (54), we see that $W_{R}^{(1)}$ does not decay to quarks and $W_{L}^{(1)}$ does not decay to $N-\tilde{\ell}$ pair. All this is what we anticipated already. For the illustrative purpose, in Fig. 3, we show the results ignoring the $W^{(0)}$ component in the mixing, which would lead to an error of the size $\frac{g_{\star W}^{2} v^{2}}{4 m_{\star}^{2}}<0.1$. From there, we see that $W_{R}$ indeed decays dominantly to $N-\tilde{\ell}$ pair, providing production mechanism for them. This can be contrasted to the case of fourdimensional LR models, where the dominant decay is into jets. For our collider study in Sec. V, however, we used full model including Higgs-induced mixing and mass splitting.
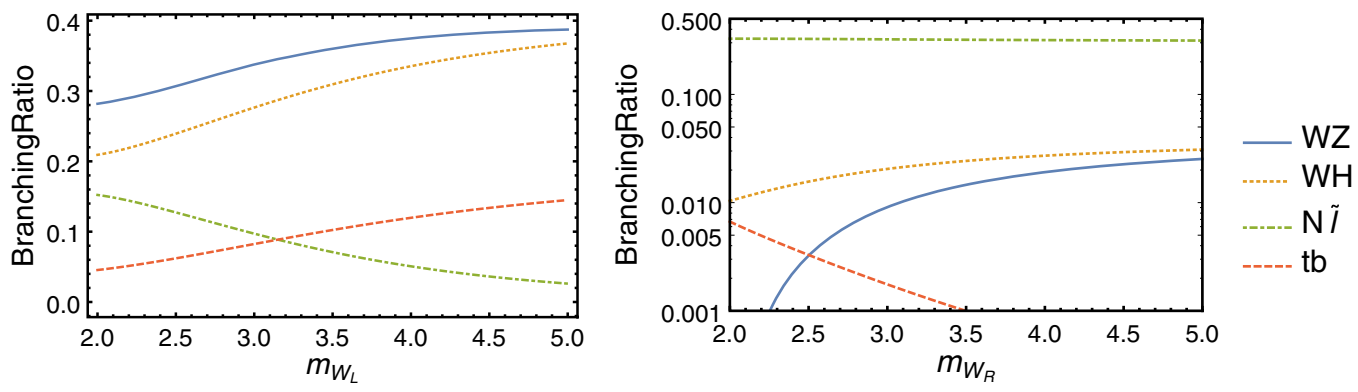

FIG. 3. The plot on the left (right) panel shows branching ratios of $W_{L}\left(W_{R}\right)$ as a function of its mass. 


\section{D. $N$ and $\tilde{\ell}$ production and decay}

As mentioned in the last section, $N$ and $\tilde{\ell}$ are produced from on-shell decay of $W_{L}$ and $W_{R}$ via couplings in Eq. (51). Decays of them are proceeded via the couplings Eq. (52), resulting in decay widths:

$$
\begin{aligned}
\Gamma(N \rightarrow W \ell) & \approx y^{2} \frac{m_{\tilde{L}_{R}}}{32 \pi} \\
\Gamma(N \rightarrow H / Z \nu) & \approx y^{2} \frac{m_{\tilde{L}_{R}}}{64 \pi} \\
\Gamma(\tilde{\ell} \rightarrow W \nu) & \approx y^{2} \frac{m_{\tilde{L}_{R}}}{32 \pi} \\
\Gamma(\tilde{\ell} \rightarrow H / Z \ell) & \approx y^{2} \frac{m_{\tilde{L}_{R}}}{64 \pi} .
\end{aligned}
$$

In principle, there will be three body decays via virtual $W_{R}$. However, we have checked that, for the choice of parameters we made, such three body decays are suppressed compared to 2 body decays.

So far, we have focused on production and decay of charged gauge bosons, $W_{L}$ and $W_{R}$, and resulting production of singlet neutrino $N$. In addition to these, however, the model also contains neutral gauge bosons $Z_{1}$ and $Z^{\prime}$ (see Sec. III). The relevant couplings for these neutral gauge bosons can be obtained in a similar way as those for charged ones. In particular, just like Eq. (50) for charged gauge bosons, $Z_{1}\left(Z^{\prime}\right)$ couplings to light quarks is basically $\frac{g_{Z}^{2}}{g_{\star Z}}$ times a factor for EWSB-induced mixing, and it is via this couplings that neutral gauge bosons are produced at the LHC. In our framework (i.e. five-dimensional/composite LR model), since $Z_{1}$ and $Z^{\prime}$ arise as composite vector mesons of the strong dynamics in the same way as the charged ones do, they have the same/comparable mass as $W_{L}\left(W_{R}\right)$. This, then, naturally leads to the comparable production rates for $Z_{1}$ and $Z^{\prime}$, i.e. they are not suppressed compared to $W_{L}$ and $W_{R}$. This feature can be contrasted to the case of four-dimensional LR models, where production of $Z^{\prime}$ is suppressed compared to $W_{R}$ due to the fact that $Z^{\prime}$, as an elementary particle, is heavier than $W_{R}$. Moving onto the decays of the neutral gauge bosons, for the same reason for the charged gauge bosons, $Z_{1}$ and $Z^{\prime}$ also have significant branching ratio to a pair of $N$. In this way, we see that, production and decay of these neutral gauge bosons provide another way to abundantly produce a pair of singlet neutrinos $N$. This signal channel, however, has almost the same process topology as four-dimensional LR. Instead, we are planning to study the production of the singlet neutrino via on-shell decay of neutral gauge boson in our follow-up paper, but in a slightly different setup with interesting features/differences that only the five-dimensional framework can furnish.

\section{DISCOVERY POTENTIAL}

In this section, we present our results for phenomenological studies of the LHC signals for the model described in Sec. III. In particular, we study the pair production of the singlet neutrino $(N)$ and its $S U(2)_{\mathrm{R}}$ partner $(\tilde{\ell})$ via the oneshell decay of $W_{R}$ and $W_{L}$, and their subsequent decays to SM particles. We consider two benchmark points depending on how $N$ and $\tilde{\ell}$ cascade decay to SM particles: Dilepton and trilepton channels.

For the dilepton channel, the production and the cascade decays of $N$ and $\tilde{\ell}$ are as follows:

$$
\begin{gathered}
p p \rightarrow W_{L} / W_{R} \rightarrow N \tilde{\ell}^{ \pm}, \quad N \rightarrow \ell^{ \pm}\left(W^{\mp} \rightarrow j j\right), \\
\tilde{\ell}^{ \pm} \rightarrow \ell^{ \pm}(H / Z \rightarrow b \bar{b}) .
\end{gathered}
$$

Hence, the final states of the dilepton channel consist of $\ell \ell j j b \bar{b}$, where, for the lepton pair, only opposite sign combination can arise since we are ignoring small Majorana splitting for $N$. That is, this process is leptonnumber conserving. In particular, this channel contains two leptons, and hence the name for the channel.

For the trilepton channel, on the other hand, we take the leptonic decay for the SM $W$ boson from $N$. In detail, we get

$$
\begin{gathered}
p p \rightarrow W_{L} / W_{R} \rightarrow N \tilde{\ell}^{ \pm}, \quad N \rightarrow \ell^{ \pm}\left(W^{\mp} \rightarrow \ell^{\mp} \nu\right), \\
\tilde{\ell}^{ \pm} \rightarrow \ell^{ \pm}(H / Z \rightarrow b \bar{b}) .
\end{gathered}
$$

Hence, the final states of the trilepton channel consist of $\ell \ell \ell \nu b \bar{b}$. This time, it contains three leptons, explaining the name of the channel.

Notice that in both channels, we add contributions from both $H$ and $Z$ decaying into $b \bar{b}$. This is because resolutions of LHC detectors may not be good enough to distinguish those two cases, and at the same time, we will achieve a slight increase in the signal rate.

The Feynman diagrams for both signal processes are shown in Fig. 4. The topology of our signal processes are characterized by several resonance peaks in various invariant mass variables. In particular, invariant masses of $W_{R}$ and $N / \tilde{\ell}$, which we take to be $M_{W_{R}}=2 \mathrm{TeV}$ and $M_{N}=$ $750 \mathrm{GeV}$ in our study, will draw sharp distinctions between signal and SM backgrounds. For the trilepton channel, however, due to the presence of neutrino and the multiplicity of leptons (i.e. combinatorics issue), naively, one would think that resonance peaks are less pronounced. However, as we show below, by reconstructing the longitudinal component of the neutrino's momentum and by figuring out the identification of each lepton, i.e., which lepton is to be paired with $b \bar{b}$, neutrino, and $\ell \nu$, respectively, we are able to construct all invariant mass peaks.

Event simulations are performed by employing a sequence of simulation tools. We first created our two-site simplified model files using FEynRules [22] based on Heavy Vector Triplets models [23]. Then we used them as inputs model in a Monte Carlo event generator MG5AMC@NLO [24] to generate parton level events. 


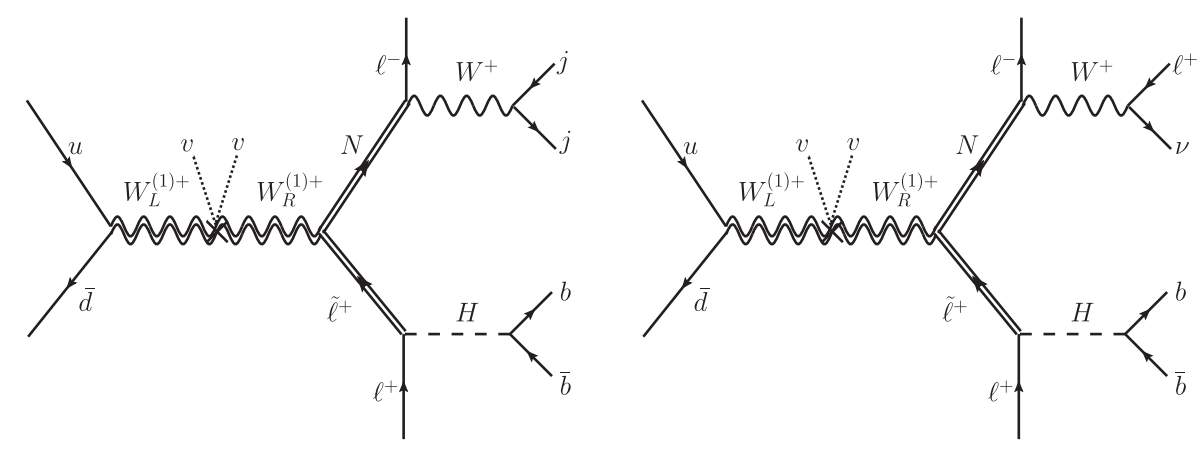

FIG. 4. The left panel shows Feynman diagram for the signal process of the dilepton channel. The right panel shows Feynman diagram for the signal process of the trilepton channel. Double (single) lines denote composite (elementary) particles. Here composite gauge bosons are in gauge basis $W_{L}^{(1)}$ and $W_{R}^{(1)}$ in order to show the mixing induced by Higgs VEV explicitly.

In this procedure, parton distribution functions parameterized by NN23LO1 [25] is used. All the simulations are done at the leading order with a $\sqrt{s}=14 \mathrm{TeV} p p$ collider. The generated parton level events are then streamlined to PYTHIA 6.4 [26] to take care of showering and hadronization/fragmentation. For our choice of parameters discussed above, in a sizable fraction of signal events, jets from hadronic decays of $W / Z / H$ are merged to form a single jet given a fixed radius. In our current study, however, we constrain ourselves to a kinematic region where the decays of electroweak bosons result into two resolved jets (or similarly charged leptons). This allows us to directly pass on the output from Pythia 6.4 to Delphes 3 [27]. DELPHES 3, interfaced with FASTJET $[28,29]$, provides a way to incorporate the detector effects and jet formation. This includes $b$-(miss)tagging and lepton/photon identifications at the detector, which usually depend on $p_{T}$ and rapidity of the object under consideration. The jets are constructed with the anti- $k_{t}$ algorithm [29] with a radius parameter $R=0.4$.

In Sec. VA, we present our results for the dilepton channel. Results for the trilepton channel follow in Sec. V B. We also briefly discuss phenomenological distinctions between our five-dimensional left-right symmetry model and that of four dimensions. In particular, we will point out several salient features of our case by which two frameworks can be distinguished once discovery is made.

\section{A. Dilepton + dijet $+H / Z$ channel}

We begin by considering the production of $N-\tilde{\ell}$ pair and their decays at the LHC. In our current study, we consider $(N, \tilde{\ell})$ as a $S U(2)_{\mathrm{R}}$ doublet and as a consequence the production of this doublet pair should be proceeded via decay of $W_{R}^{(1)}$ gauge boson. However, since SM quarks are not charged under $S U(2)_{\mathrm{R}}$ gauge group, $W_{R}^{(1)}$ can only be produced via its mixing with $W_{L}^{(1)}$. Namely, once $W_{L}^{(1)}$ is produced via quark fusion inside the proton through its
$S U(2)_{\mathrm{L}}$-coupling, EWSB-induced mixing between $W_{L}^{(1)}$ and $W_{R}^{(1)}$ leads to the production of $W_{R}^{(1)} \cdot W_{R}^{(1)}$ then subsequently decays into $N-\tilde{\ell}$ pair. As shown in Sec. III, the size of $W_{R}^{(1)}-W_{L}^{(1)}$ mixing angle is $\tan 2 \theta_{23} \approx$ $\frac{1}{2} g_{\star W}^{2} v^{2} /\left(M_{W_{L}^{(1)}}^{2}-M_{W_{R}^{(1)}}^{2}\right)$ [see Eq. (34)], and when the mass splitting, $M_{W_{L}^{(1)}}^{2}-M_{W_{R}^{(1)}}^{2}$, is small enough we acquire significant mixing, leading to enhanced production for signal. This can be realized when the masses of $W_{L}^{(1)}$ and $W_{R}^{(1)}$ are approximately degenerate and the mass scale itself is low enough. Motivated by the consistency with the electroweak precision measurements (EWPM), our fivedimensional warped extra-dimensional seesaw model or its two-site simplified model has built-in left-right symmetries, allowing desired mass degeneracy. In addition, the consistency with EWPM permits the mass of $M_{W_{L}^{(1)}} / M_{W_{R}^{(1)}}$ as low as $\mathcal{O}(2) \mathrm{TeV}$. Such a low mass for $W_{L}^{(1)} / W_{R}^{(1)}$ further allows, in addition to large mixing, resonance enhancement for the signal production cross section at the LHC. Moving onto the decay of $W_{R}^{(1)}$, as elaborated in Sec. IVC, it will dominantly decay into $(N, \tilde{\ell})$ pair. Therefore, making use of all these features, we can secure enough statistics for signal production at $14 \mathrm{TeV}$ LHC. In the dilepton channel, $N$ decays to $W^{ \pm} \ell^{\mp}$ and $\mathrm{SM} W$ boson, in turn, decays hadronically producing two jets. On the other hand, $\tilde{\ell}^{ \pm}$ decays to $\ell^{ \pm} H / Z$, which is then followed by decay of $H / Z$ to $b \bar{b}$. As is evident from these cascade decays of $N$ and $\tilde{\ell^{ \pm}}$, (i) signal process does not contain any neutrinos and hence no missing energy and (ii) there are several invariant mass variables which are all fully reconstructible. Those invariant mass variables include, $M_{j j}, M_{b \bar{b}}, M_{j j \ell}, M_{b \bar{b} \ell}$, and $M_{\text {All }}$, where $M_{\text {All }}$ is the invariant mass of all reconstructed/ visible particles. If successfully reconstructed, for signal, the distributions of these variables will be peaked at $M_{W}$, $M_{H / Z}, M_{N}, M_{N}$, and $M_{W_{R}}$, respectively.

There are several SM backgrounds we need to consider and we describe them one by one now. 
(1) $t \bar{t} j j$ : The relevant process is $p p \rightarrow t \bar{t} j j \rightarrow$ $\ell^{-} \ell^{+} \nu \bar{\nu} b \bar{b} j j$, where $t \rightarrow b\left(W^{+} \rightarrow \ell^{+} \nu\right)$, and similarly for $\bar{t}$, is considered. Being a purely QCD process, this is the background with largest cross section. Background reduction will be achieved by means of a combination of various invariant mass cuts. Particularly useful ones will be $M_{\text {All }}$ and $M_{b \bar{b} \ell} / M_{j j \ell}$ cuts. In principle, missing transverse momentum $E_{T}$, the opposite of the vectorial $p_{T}$ sum of reconstructed objects in the event, can provide useful reduction, although we found other cuts are more efficient. It may be worthwhile to mention that in principle totally inclusive $t \bar{t} j j$ process will include $p p \rightarrow t \bar{t} W^{ \pm}, W^{ \pm} \rightarrow \ell^{ \pm} \nu(\bar{\nu})$. In fact, one can easily check that pure QCD-initiated $t \bar{t} j j$ and $t \bar{t} W$-initiated processes are parametrically the same order. However, MG5AMC@NLO simulation of $t \bar{t} j j$ does not include $t \bar{t} W$, partly due to a slightly different (but equivalent) power counting adopted. Numerically, we found that the cross section for the $t \bar{t} W$-initiated process $(\approx 7.5 \mathrm{fb})$ is much smaller than that of the $t \bar{t} j j$-initiated process $\left(\approx 2 \times 10^{4} \mathrm{fb}\right)$, and hence we do not include $t \bar{t} W$ background in our analysis.

(2) $t \bar{t} H / Z$ : The relevant process is $p p \rightarrow t \bar{t} H / Z \rightarrow$ $\ell^{-} \ell^{+} \nu \bar{\nu} b b \bar{b} \bar{b}$, where $t \rightarrow b\left(W^{+} \rightarrow \ell^{+} \nu\right)$, and similarly for $\bar{t}$, and $H / Z \rightarrow b \bar{b}$ are considered. If two $b$ 's in the signal process are b-tagged as a part of selection criteria, then in order for this background to pass the selection criteria, two of four $b$ 's must be untagged as regular two jets, leading to a large reduction of the background. Moreover, $M_{\text {All }}$, $M_{b \bar{b} \ell} / M_{j j \ell}$ and $M_{j j}$ cuts will be useful.

(3) $j j \ell \ell H / Z$ : The relevant process is $p p \rightarrow$ $j j \ell^{-} \ell^{+} H / Z, H / Z \rightarrow b \bar{b}$, where the lepton pair comes mostly from decay of on-shell $Z$ (and offshell photon). Therefore, in this process, the distribution of the dilepton invariant mass, $M_{\ell \ell}$, will be sharply peaked at the mass of the $Z$ boson, $M_{Z}$. However, since two leptons in the signal process do not reconstruct $M_{Z}$, the condition $M_{\ell \ell} \neq M_{Z}$ will remove most of this background. In addition, $M_{\mathrm{All}}$, $M_{b \bar{b} e} / M_{j j e}$ and $M_{j j}$ cuts will be useful.

(4) irred (irreducible background): The relevant process is $p p \rightarrow \ell^{-} \ell^{+} W^{ \pm} H / Z, W^{ \pm} \rightarrow j j, H / Z \rightarrow b \bar{b}$. Similarly to $j \mathrm{\ell} \ell \ell H / Z$ background, the lepton pair will mostly arise from the on-shell decay of $Z$ and the cut $M_{\ell \ell} \neq M_{Z}$ will significantly reduce this events. Even though $j j(b \bar{b})$ will successfully reconstruct $M_{W}\left(M_{H / Z}\right), M_{\text {All }}$ and $M_{b \bar{b} \ell} / M_{j j \ell}$ cuts will still provide additional significant reduction of this background events.

Defining $N_{\ell}, N_{b}$ and $N_{j}$ as the number of isolated leptons, b-tagged jets, and non-b-tagged jets, respectively, we select events using the following selection criteria:

$$
\begin{array}{lll}
N_{\ell}>1 & \text { with } & \left|\eta_{\ell}\right|<2.5 \\
N_{b}>1 & \text { with } & \left|\eta_{b}\right|<3 \\
N_{j}>1 & \text { with } & \left|\eta_{j}\right|<3 .
\end{array}
$$

In addition, we impose a set of basic cuts $p_{T j} / p_{T b}>$ $20 \mathrm{GeV}$ and $p_{T \ell}>10 \mathrm{GeV}$ at parton level event simulation, partly to avoid possible IR-divergence issues for background simulations. We reimpose such cuts on objects (hardest two jets, two $b$-jets, and two leptons) that pass selection criteria of Eq. (58). We use $p_{T}$ to evaluate hardness of the reconstructed objects and take the hardest two. In Fig. 5, we show distributions of various variables for signal and background events that pass selection criteria and basic cuts. In particular, we see that $M_{\text {All }}$ (top row, left), the invariant mass of all reconstructed objects, i.e. hardest two $j$ 's + two $b$ 's + two $\ell$ 's, for signal is peaked at $2 \mathrm{TeV}$, the mass of $W_{R}$ we take, and is well separated from all backgrounds, providing a strong cut to reduce backgrounds. Similar sharp distinctions are drawn for $M_{j j \ell}$ (mid row, right) and $M_{b \bar{b} \ell}$ (mid row, left), but with slightly larger overlap with backgrounds. These two variables reconstruct the mass of $N$ and $\tilde{\ell}$, respectively. It may be worth describing the way we reconstruct these variables. The subtlety might be that since there are two leptons in the final states, it would be crucial to figure out which lepton is to be paired with the $b$-pair, and similarly for the $j$-pair. We found, for example, that naively plotting the invariant mass of the $b$-pair with both leptons (similarly $j$-pair with both leptons) does not reveal the sharp peak at $M_{N}$, and the resulting distribution is broadly extended with large overlap with background distributions. In order to achieve sharper distinction, we make use of the fact that the masses of $N_{R}$ and $\tilde{\ell}_{R}$ are equal due to $S U(2)_{\mathrm{R}}$ invariance. Namely, we identify the lepton that goes with the $b$-pair $\left(\ell_{b}\right)$ and the one that goes with the $j$-pair $\left(\ell_{j}\right)$ by minimizing

$$
\left|M_{b \bar{b} \ell_{b}}-M_{j j \ell_{j}}\right| .
$$

As can be seen from Fig. 5, this criterion successfully reconstructs $M_{N}$ for majority of events, albeit imperfect. In this way, both $M_{b \bar{b} \ell}$ and $M_{j j \ell}$ provide another set of very useful cuts. Next very useful variable is $M_{\ell \ell}$ (top row, right). As anticipated above while we discuss each backgrounds, $M_{\ell \ell}$ distributions for $j j \ell \ell H / Z$ and irred backgrounds are sharply localized at $M_{Z}$. In addition, other backgrounds also tend to be distributed over smaller $M_{\ell \ell}$ values compared to signal (see the inset plot of $M_{\ell \ell}$ distribution of Fig. 5). The bottom row of Fig. 5 shows $M_{j j}, M_{b \bar{b}}$, and $E_{T}$ distributions. We see that the $M_{j j}\left(M_{b \bar{b}}\right)$ distribution for signal events develops a peak at $M_{W}$ $\left(M_{H / Z}\right)$ as expected. This is not true, on the other hand, for two major backgrounds: $t \bar{t} j j$ and $t \bar{t} H / Z$. Therefore, these variables will supplement the above-described variables to attain additional suppression of background events. 

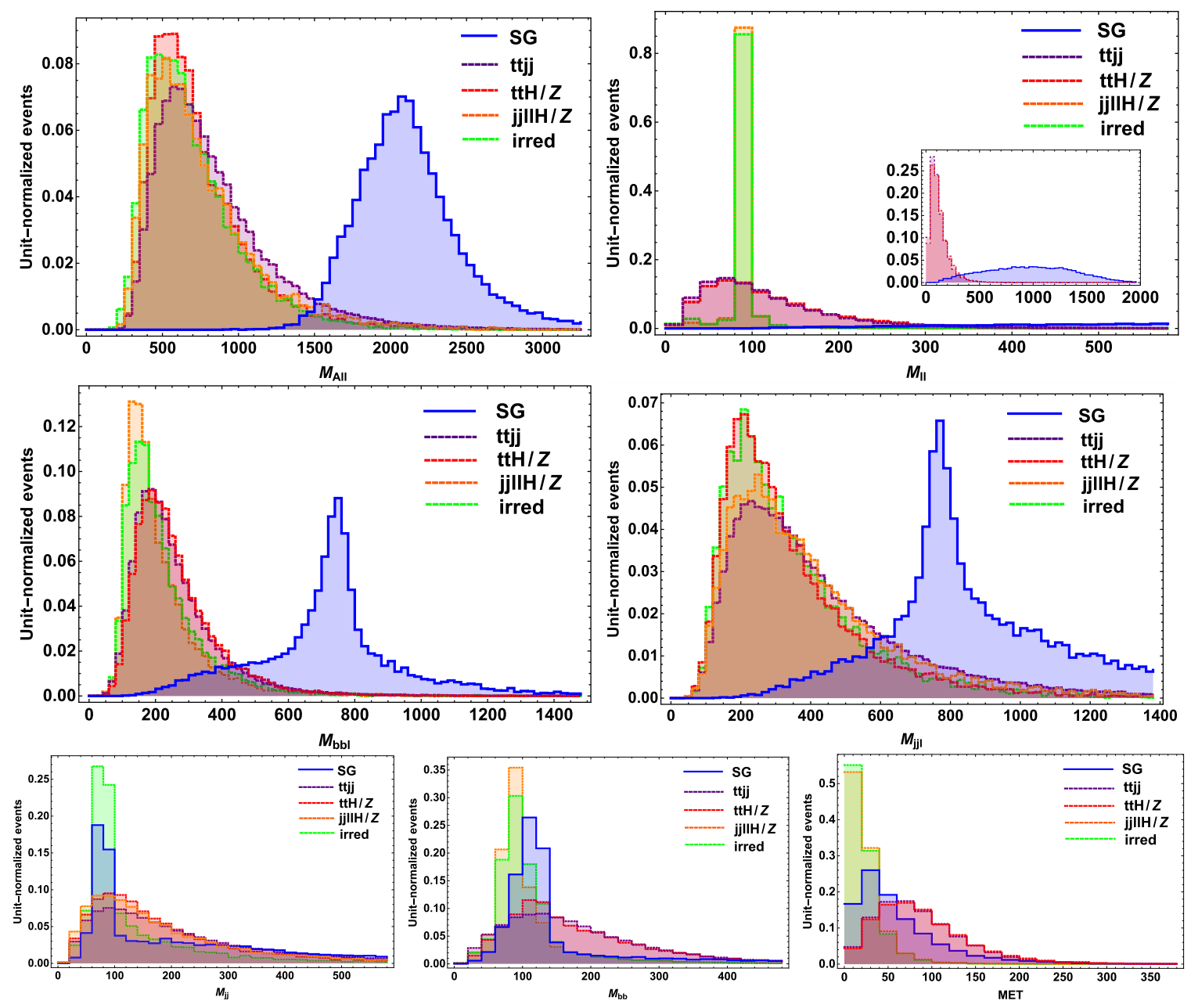

FIG. 5. Dilepton channel: Distributions of variables: $M_{\mathrm{All}}$ (top row, left), $M_{\ell \ell}$ (top row, right), $M_{b \bar{b} \ell}$ (mid row, left), $M_{j j \ell}$ (mid row, left), $M_{j j}$ (bottom row, left), $M_{b \bar{b}}$ (bottom row, mid) and $\operatorname{MET}\left(\mathscr{E}_{T}\right)$ (bottom row, right) for signal (solid blue) and backgrounds (dotted, $t \bar{t} j j$-purple, $t \bar{t} H / Z$-red, $j j \ell \ell H / Z$-orange, irred-green).

Finally, the missing transverse momentum variable also helps a bit. This is expected based on the insight that the backgrounds $t \bar{t} j j$ and $t \bar{t} H / Z$ have larger $E_{T}$ than signal. We provide the cut flows for the signal and the major SM backgrounds in Table I. We find that the dilepton channel may provide a sensitivity to uncover the warped seesaw nature by $\sim 3.5 \sigma$ with an integrated luminosity of $\mathcal{L}=$ $300 \mathrm{fb}^{-1}$ and even by $\sim 11 \sigma$ with $\mathcal{L}=3000 \mathrm{fb}^{-1}$.

\section{B. Trilepton $+H / Z$ channel}

In this section, we present the results for the trilepton channel. Similarly to the dilepton channel discussed in the previous section, the $N-\tilde{\ell}$ pair is produced via the decay of $W_{R}^{(1)}$ using large mixing between $W_{R}^{(1)}$ and $W_{L}^{(1)}$. In the trilepton channel, $N$ decays to $W^{ \pm} \ell^{\mp}$ and SM $W$ boson, in turn, decays leptonically producing $\ell \nu$. Like in the dilepton channel, $\tilde{\ell}^{ \pm}$decays to $\ell^{ \pm} H / Z$, with subsequent decay of $H / Z$ to $b \bar{b}$. As is evident from these cascade decays of $N$ and $\tilde{\ell}^{ \pm}$, (i) the signal process now does contain a neutrino, leading to missing energy and (ii) there are three leptons in final states. The existence of the neutrino (or missing particle in general) and the large multiplicity of leptons can be a potential obstacle in reconstruction of resonance peaks. However, we will show below that such difficulty can be, at least partly, overcome by reconstruction of longitudinal momentum of neutrino and by cleverly figuring out lepton identifications. Once these are done, various invariant mass variables can be successfully reconstructed and used to reduce backgrounds. Those invariant mass variables include $M_{\ell \nu}, M_{b \bar{b}}, M_{\ell \ell \nu}, M_{b \bar{b} \ell}$, and $M_{W_{R}}^{\text {recon }}$, where $M_{W_{R}}^{\text {recon }}$ is the invariant mass constructed from all reconstructed visible particles and reconstructed neutrino four momentum. When properly reconstructed, the signal distribution of these variables will be peaked at $M_{W}, M_{H / Z}$, $M_{N}, M_{N}$, and $M_{W_{R}}$, respectively. Additional invariant mass variables exist: $M_{\ell \ell}, M_{\ell \ell \ell}$, and $M_{\mathrm{All}}$, where $M_{\mathrm{All}}$ is the invariant mass of all reconstructed/visible particles without neutrinos. These variables do not correspond to any of the 
TABLE I. Cut flows for signal and major background events in terms of their cross sections. The cross sections are in fb. The numbers in the first row ("generator-level cuts") are cross sections obtained with basic cuts at the generation level to avoid divergence (for both signal and backgrounds). In the second row, the same basic cuts are reimposed to both signal and background events along with multiplicity requirements for b-jet, non-b-jet, and leptons. Once the cross section decreases such that the net number of events at $\mathcal{L}=3000 \mathrm{fb}^{-1}$ is less than 1 , we report it as " $0 . "$

\begin{tabular}{lccccc}
\hline \hline Cuts & Signal & $t \bar{t} j j$ & \multicolumn{1}{c}{$t \bar{t} H$} & $j j \ell \ell H / Z$ irred \\
\hline Generator-level cuts & 0.73 & $18.2 \times 10^{3}$ & 18.1 & 46.8 & 0.32 \\
$N_{\ell}>1, N_{j}>1, N_{b}>1$ & 0.12 & $2.0 \times 10^{3}$ & 3.11 & 3.97 & 0.030 \\
$\quad$ with basic cuts & & & & & \\
$M_{\ell \ell} \in[400, \infty] \mathrm{GeV}$ & 0.11 & 25.6 & 0.045 & 0.0094 & 0 \\
$M_{\text {All }} \in[1600, \infty] \mathrm{GeV}$ & 0.11 & 6.50 & 0.01 & 0.0028 & 0 \\
$M_{b \bar{b}} \in[0,200] \mathrm{GeV}$ & 0.09 & 2.04 & 0.0034 & 0.0014 & 0 \\
$M_{b \bar{b} \ell} \in[550, \infty] \mathrm{GeV}$ & 0.07 & 0.055 & 0.00091 & 0.00047 & 0 \\
$\mathbb{E}_{T} \in[0,100] \mathrm{GeV}$ & 0.056 & 0.018 & 0.00072 & 0.00047 & 0 \\
$S / B$ & 2.92 & $\ldots$ & $\ldots$ & $\ldots$ & $\ldots$ \\
$S / \sqrt{S+B}$ & 3.54 & $\ldots$ & $\ldots$ & $\ldots$ & $\ldots$ \\
$\quad\left(\mathcal{L}=300 \mathrm{fb}^{-1}\right)$ & & & & & \\
$S / \sqrt{S+B}$ & 11.2 & $\ldots$ & $\ldots$ & $\ldots$ & $\ldots$ \\
$\left(\mathcal{L}=3000 \mathrm{fb}^{-1}\right)$ & & & & & \\
\hline \hline
\end{tabular}

resonance peaks in the signal process. However, they will still provide very strong distinctions between the signal and backgrounds.

There are several SM backgrounds we need to consider, and we describe them one by one now.

(1) $t \bar{t} W$ : The relevant process is $p p \rightarrow t \bar{t} W^{ \pm} \rightarrow$ $\ell^{-} \ell^{+} \ell^{ \pm} \nu \bar{\nu} \nu(\bar{\nu}) b \bar{b}$, where $t \rightarrow b\left(W^{+} \rightarrow \ell^{+} \nu\right)$, and similarly for $\bar{t}$, is considered. All SM $W$ 's decay leptonically: $W^{ \pm} \rightarrow \ell^{ \pm} \nu(\bar{\nu})$.

(2) irred (irreducible background): The relevant process is $p p \rightarrow \ell^{-} \ell^{+} W^{ \pm} H / Z, W^{ \pm} \rightarrow l^{ \pm} \nu(\bar{\nu}), H / Z \rightarrow b \bar{b}$.

(3) $\ell^{-} \ell^{+} W j j$ : The relevant process is $p p \rightarrow$ $\ell^{-} \ell^{+} W^{ \pm} j j, W^{ \pm} \rightarrow l^{ \pm} \nu(\bar{\nu})$. Since we will select events with two $b$ 's are tagged, only very small fraction of events with two regular jets mistagged as b-tagged jets will contribute to the backgrounds. Mistag rate is typically $\lesssim 1 \%$ [30] and $u d s$-jet mistag rate can even be as small as $0.3 \%$ [31]. The cross section of the process is $\sigma \sim 180 \mathrm{fb}$ and the surviving events with two mistagging is $\sim \mathcal{O}(0.01) \mathrm{fb}$. This corresponds to roughly $\sim \mathcal{O}(3)$ events at an integrated luminosity of $\mathcal{L}=300 \mathrm{fb}^{-1}$. It will be very unlikely that any of these events will in the signal region given the number of invariant mass cuts that it should pass. Hence, we will not explicitly consider this background for our analysis.

Defining $N_{\ell}$ and $N_{b}$ as the number of isolated leptons and b-tagged jets, respectively, we select events using the following selection criteria:

$$
\begin{array}{lll}
N_{\ell}>2 & \text { with } & \left|\eta_{\ell}\right|<2.5 \\
N_{b}>1 & \text { with } & \left|\eta_{b}\right|<3 .
\end{array}
$$

In addition, we impose a set of basic cuts $p_{T b}>20 \mathrm{GeV}$ and $p_{T \ell}>10 \mathrm{GeV}$ at parton level event simulation, partly to avoid possible IR-divergence issues for background simulations. We reimpose such cuts on objects (hardest two $b$-jets, and three leptons) that pass selection criteria of Eq. (60). We use $p_{T}$ to evaluate hardness of the reconstructed objects.

Next, we discuss the way we reconstruct longitudinal component of neutrino's four momentum. Together, we also discuss how we figure out lepton identifications. Namely, we want to know, out of three leptons selected as described above, which one is produced together with $b \bar{b}$ from the decay of $\tilde{\ell}^{ \pm}$(we call it $\ell_{b}$ ) and which one is produced directly from the decay of $N$ (we call it $\ell_{W}$ ), and finally which one is the decay product of SM $W$ (we call it $\ell_{\nu}$ ). ${ }^{26}$ First of all, for a given choice of lepton (a candidate for $\ell_{\nu}$ ), the $z$ component of the neutrino's momentum can be obtained by requiring

$$
M_{W}^{2}=\left(p_{\mu}^{\nu}+p_{\mu}^{\ell}\right)^{2},
$$

where $M_{W}$ is the mass of the SM $W$ boson. For $p_{\mu}^{\nu}$, we use the fact that neutrino is massless, $\left(p_{\mu}^{\nu}\right)^{2}=0$. Then, the above equation is a quadratic equation for the $z$ component of $p_{\mu}^{\nu}$, and if solutions exist, there are two solutions, unless determinant vanishes by numerical coincidence. In this case, we pick up $p_{z}^{\nu}$ that minimizes the sum of the $z$ component of all particles' momenta, i.e. the sum of $p_{z}$ of two $b$ 's, three $\ell$ 's, and $\nu$. This is based on the insight that $W_{R}$ is mostly produced at rest. In case when the determinant of the quadratic equation is less than 0 , so that no solution exists, we set

$$
p_{z}^{\nu}=-\sum_{\text {all visible }} p_{z}
$$

This choice again is motivated by the intuition that $W_{R}$ is mostly produced at rest. Once the $z$ component of the neutrino's momentum (or equivalently full $p_{\mu}^{\nu}$ ) is reconstructed this way for a given choice of $\ell$ (again a candidate for $\ell_{\nu}$ ), we then determine $\ell_{b}$ and $\ell_{W}$ by minimizing

$$
\left|M_{b \bar{b} \ell_{b}}-M_{\ell_{\nu} \ell_{W} \nu}\right| \cdot
$$

This criteria is motivated as before by $S U(2)_{\mathrm{R}}$ invariance and resulting mass degeneracy. In this way, for each choice of $\ell_{\nu}$, we determine full $p_{\mu}^{\nu}$ and identify, for the remaining two leptons, which lepton is $\ell_{b}$ and which lepton is $\ell_{W}$. We repeat this procedure for all three possible choices of

\footnotetext{
${ }^{26}$ The subscript is designed to indicate a set of particles that the lepton accompanies.
} 

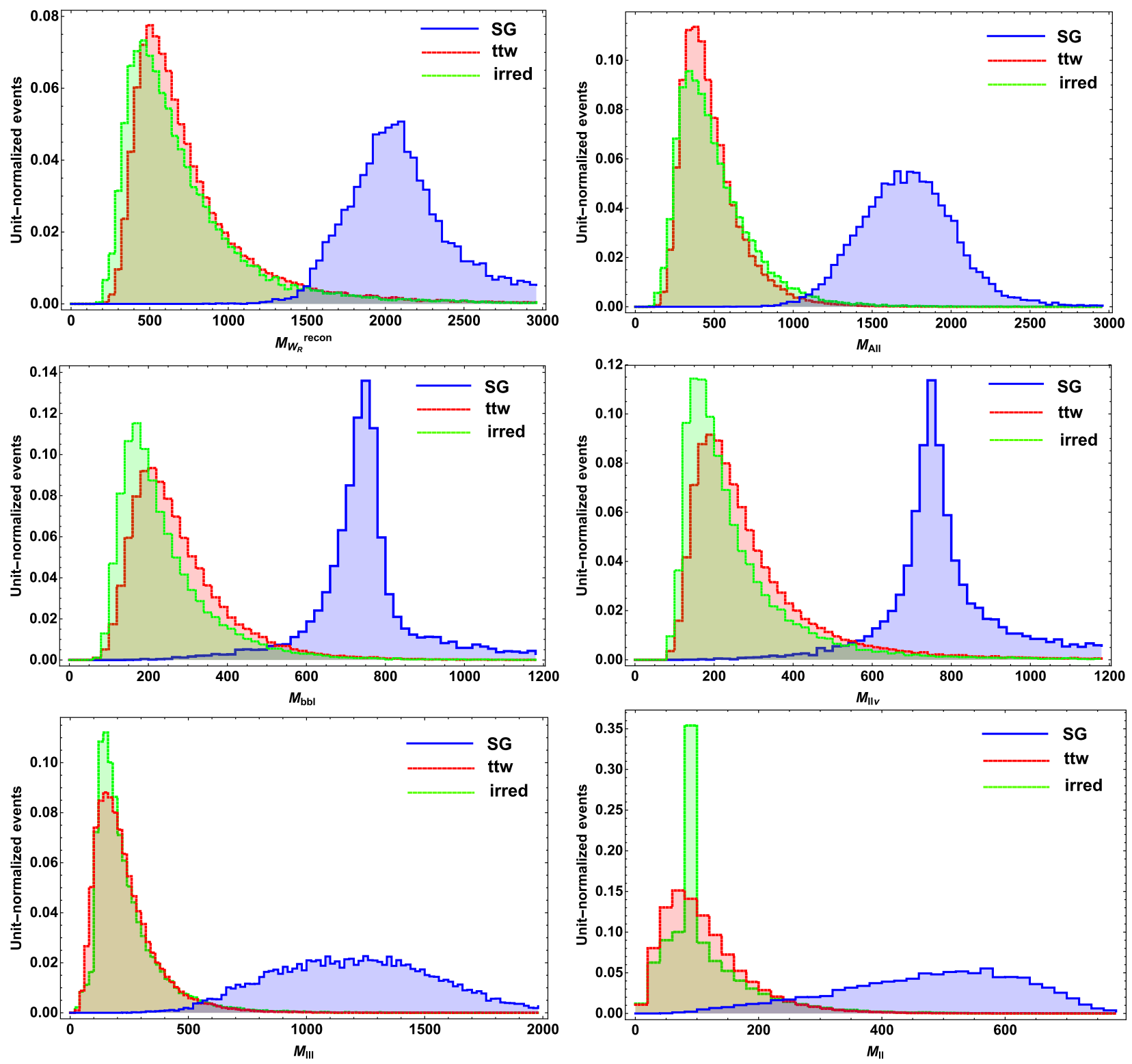

FIG. 6. Trilepton channel: Distributions of variables: $M_{W_{R}}^{\text {recon }}$ (top row, left), $M_{\text {All }}$ (top row, right), $M_{b \bar{b} \ell}$ (mid row, left), $M_{\ell \ell \nu}$ (mid row, right), $M_{\ell \ell \ell}$ (bottom row, left), and $M_{\ell \ell}$ (bottom row, right) for signal (solid blue) and backgrounds (dotted, $t \bar{t} W$-red and irred-green).

$\ell_{\nu}$. Final decision is made for the combination $\left\{\ell_{\nu}, \ell_{W}, \ell_{b}\right\}$ that renders minimum value for Eq. (63). In Fig. 6, we show distributions of various invariant mass variables for signal and background events that pass selection criteria and basic cuts. These invariant mass variables are constructed using $\left\{\ell_{\nu}, \ell_{W}, \ell_{b}\right\}$-identification and full $p_{\mu}^{\nu}$ reconstructed as described above. In particular, in addition to $M_{\text {All }}, M_{\ell \ell}$, $M_{b \bar{b}}$ and $M_{\ell \ell \ell}$, which are all possible without knowing detailed information about lepton identification and full momentum for neutrino, now we can also explicitly compute $M_{W_{R}^{\text {recon }}}, M_{b \bar{b} \ell_{b}}$, and $M_{\ell_{\nu} \ell_{W} \nu}$. These latter variables would not be possible without figuring out lepton identification, i.e. $\left\{\ell_{\nu}, \ell_{W}, \ell_{b}\right\}$, and full momentum for neutrino. To be more precise, we can actually calculate $M_{b \bar{b} \ell_{b}}$ and
$M_{\ell_{\nu} \ell_{W \nu}}$ by simply considering all possible combination of two leptons, and we found that such computed distributions do not reveal any sharp peak and rather show very broad distributions, failing to provide strong cuts to reduce background. In Fig. 6, we show distributions of $M_{b \bar{b}} \ell_{b}$ (mid row, left) and $M_{\ell_{\nu} \ell_{W}}$ (mid row, right). Both distributions are sharply peaked at $750 \mathrm{GeV}$, a input value for $M_{N}$. In the case of $M_{W_{R}^{\text {recon }}}$, we really need to know full $p_{\mu}^{\nu}$ to be able to compute it. The left panel of the top row in Fig. 6 shows $M_{W_{R}^{\text {recon }}}$ distribution and it is indeed peaked at $/$ near $2 \mathrm{TeV}$, a input value for $W_{R}^{(1)}$. This is to be compared to the $M_{\text {All }}$ distribution shown in the right panel of the top row in Fig. 6. Again, $M_{\text {All }}$ is the invariant mass for all 
TABLE II. Cut flows for signal and major background events in terms their cross sections. The cross sections are in $\mathrm{fb}$. The numbers in the first row ("generator-level cuts") are cross sections obtained with basic cuts at the generation level to avoid divergence (for both signal and backgrounds). In the second row, the same basic cuts are reimposed to both signal and background events along with multiplicity requirements for b-jets and leptons. Once the cross section decreases such that the net number of events at $\mathcal{L}=3000 \mathrm{fb}^{-1}$ is less than 1 , we report it as " 0 ".

\begin{tabular}{lcll}
\hline \hline Cuts & Signal & $t \bar{t} W$ & \multicolumn{1}{c}{ irred } \\
\hline Generator-level cuts & 0.40 & 2.50 & 0.12 \\
$N_{\ell}>2, N_{b}>1$ with basic cuts & 0.057 & 0.30 & 0.011 \\
$M_{W_{R}^{\text {recon }} \in[1400, \infty] \mathrm{GeV}}$ & 0.057 & 0.022 & 0.00074 \\
$M_{\text {All }} \in[1000, \infty] \mathrm{GeV}$ & 0.056 & 0.0040 & 0 \\
$M_{\ell \ell \ell} \in[500, \infty] \mathrm{GeV}$ & 0.056 & 0.0030 & 0 \\
$S / B$ & 18.67 & $\ldots$ & $\ldots$ \\
$S / \sqrt{S+B}\left(\mathcal{L}=300 \mathrm{fb}^{-1}\right)$ & 3.99 & $\cdots$ & $\ldots$ \\
$S / \sqrt{S+B}\left(\mathcal{L}=3000 \mathrm{fb}^{-1}\right)$ & 12.63 & $\cdots$ & $\ldots$ \\
\hline \hline
\end{tabular}

reconstructed visible particles, i.e. two $b$ 's and three $\ell$ 's, but without neutrino. Although $M_{\text {All }}$ distribution also develops a peak with good separation from background distributions [dotted red (ttw) and dotted green (irred)], the position of the peak is shifted toward the smaller value, reflecting the existence of neutrino. We found that both $M_{W_{R}^{\text {recon }}}$ and $M_{\text {All }}$, separately, provide very efficient cuts. Overall, we see that above described prescription for reconstructing $\left\{\ell_{\nu}, \ell_{W}, \ell_{b}\right\}$-identification and full $p_{\mu}^{\nu}$ is very effective and successful. We also note that $M_{\ell \ell}$ distribution for irred is sharply peaked at $M_{Z}$ showing that two leptons come from on-shell decay of $Z$ boson. Finally, $M_{\ell \ell \ell}$-distribution for backgrounds are clustered for smaller values and well separated from that of signal. We provide the cut flows for signal and the major SM backgrounds in Table II. We find that the trilepton channel may provide a sensitivity to discover $N, \tilde{\ell}$ and $W_{R}$ by $\sim 4 \sigma$ with an integrated luminosity of $\mathcal{L}=300 \mathrm{fb}^{-1}$ and even by $\sim 13 \sigma$ with $\mathcal{L}=3000 \mathrm{fb}^{-1}$.

\section{Contrasting LHC signals with other seesaw models}

We close our discussion by pointing out several phenomenological features that can draw a distinction between the LHC signals for the five-dimensional/composite LR seesaw models studied here and other models with a similar, i.e., inverse, seesaw. ${ }^{27}$ We begin with a contrast with four-dimensional LR models, ${ }^{28}$ which perhaps have the closet resemblance to our five-dimensional setup: like in five dimensions, this four-dimensional model

\footnotetext{
${ }^{27}$ Note that distinguishing our signals from those in usual/ Majorana seesaw is more straightforward, since latter models can give same-sign dileptons from Majorana singlet neutrino production/decay vs opposite-sign from pseudo-Dirac singlets here.

${ }^{28}$ For distinguishing between various 4D seesaw models, see, for example, Ref. [32].
}

incorporates a type I seesaw; i.e., the right-handed neutrino is a singlet of $\mathrm{SM}$, in particular, $S U(2)_{L}$, albeit being charged under the extended gauge group, i.e., a doublet of $S U(2)_{R}$.

(1) First of all, the production of $W_{R}^{ \pm}$in four-dimensional LR models is via the unsuppressed coupling to quarks, whereas in the case of five-dimensional LR, it is via suppressed/smaller couplings, leading to smaller production rate.

(2) For five-dimensional/composite LR, the production of $N$ via the decay of $W_{R}^{ \pm}$accompanies its $S U(2)_{\mathrm{R}}$ partner $\tilde{\ell}$. This, in turn, renders additional Higgs/Z. Therefore, in five-dimensional/composite LR models, there are two extra resonance bumps, those of $\tilde{\ell}$ and Higgs $/ Z$. Both structures were crucial in reducing background. Perhaps more importantly, once discovery is made, these extra resonance peaks will be critical in discriminating four-dimensional vs five-dimensional LR nature.

(3) The distribution of the dilepton invariant mass will have (i) different shape and (ii) different dependence of endpoints on $M_{W_{R}^{ \pm}}$and $M_{N}$. To be more specific, for usual four-dimensional LR, the signal process is two-step cascade decay, leading to smooth distribution, except perhaps at the end point, where, depending on spin correlations, there could be a sharp/ "vertical" drop [33]. For five-dimensional/composite LR, on the other hand, having heavy $\tilde{\ell}$, in addition to $N$, in the decay of $M_{W_{R}^{ \pm}}$, the shape of the distribution will be that of an antler with a cusp, i.e., a derivative discontinuity, in roughly the middle of the distribution [34]. The end point for four-dimensional LR is located at $\sim \sqrt{M_{W_{R}}^{2}-M_{N}^{2}}$, that of five-dimensional LR being different from this.

(4) Finally, the production of $W_{R}^{ \pm}$is initiated by lefthanded quarks in our model (since it has to proceed via mixing of composite $W_{R}$ and $W_{L}$ : see Fig. 4) vs right-handed quarks for the usual LR models. Such a chiral structure of the couplings will result in different angular distributions for the singlet neutrino (and composite charged lepton) than in four-dimensional LR models. Thus, once we reconstruct these new fermionic resonances from their decays, such an analysis (in principle) could provide additional distinction between the two scenarios. However, note that such a parton-level effect tends to get washed out at a (unpolarized) $p p$ collider such as LHC, the reason being that the quark (or anti-quark) involved in the reaction is equally likely to come from either beam (cf. at a $p \bar{p}$ collider such as the TeVatron). Hence, a more sophisticated analysis would be required in order to disentangle this difference in chiral structure: we leave it for future study.

Next, we consider the type III seesaw: here, we will be more brief than above. First, take the four-dimensional version; i.e., we add a $S U(2)_{L}$ triplet, denoted by $\Sigma$ : the 
right-handed neutrino (i.e., responsible for seesaw) is the electrically neutral component, $\Sigma^{0}$, but it comes along with a new fermion, i.e., its charged partner $\Sigma^{ \pm}$. There is no extended gauge group here so that spin-1 resonance structure in the LHC signals is absent, unlike in LR models (whether four-dimensional or five-dimensional). However, suppose we make the spin-1 states heavy; i.e., we are left only with extra fermions relative to the SM. In this case, we find that there are some similarities between the LHC signals of the two models, i.e., our five-dimensional type I and type III seesaw in four dimensions. Indeed, both have new SMcharged TeV-mass fermions; in particular, our model contains $\tilde{\ell}$, the $S U(2)_{R}$ partner of the actual agent of the seesaw $(N)$, which has gauge quantum numbers of the SM righthanded charged lepton. Hence, $\tilde{\ell}$ can be pair-produced via SM $\gamma / Z$ exchange, followed by decay of $\tilde{\ell}$ into SM lepton and Higgs bosons, just like for the case of $\Sigma^{ \pm}$[again, the $S U(2)_{L}$ partner of $\Sigma^{0}$ (latter's exchange generating the SM neutrino mass)] in the type III seesaw. Yet, we might be able to distinguish some of the LHC signals that we consider from those in the type III seesaw (as follows), again, even though the spectrum of new fermions might look (roughly) similar. For example, in the type III seesaw, SM $W$ exchange can produce $\Sigma^{ \pm}$in association with $\Sigma^{0}$. Whereas, $\tilde{\ell}$ and $N$ in our model are $S U(2)_{L}$ singlets [albeit doublet of $S U(2)_{R}$ ]; they can only acquire a coupling to SM $W$ via the latter's admixture of $W_{R}$, which however is negligible in the limit of gauge KK being heavy, thus so is the $\tilde{\ell}-N$ production.

Finally, one can simply embed the type III into the fivedimensional (warped)/composite Higgs framework (along the lines of type I that we have right now) as follows. We can add a $S U(2)_{L}$ triplet (denoted as usual by $\Sigma$ ) in the bulk [instead of the SM singlet, but $S U(2)_{R}$ doublet that we have in the type I case], with a Majorana mass term on the Planck brane and coupling to Higgs and lepton doublets on the $\mathrm{TeV}$ brane. The $K K$ modes of the electrically neutral component of $\Sigma$ (denoted by $\Sigma^{0}$ ) will (effectively) give rise to an inverse seesaw mechanism (just like by $N$ in current model). Moreover, there will be LHC signals from its charged partners $\left(\Sigma^{ \pm}\right)$produced (in pairs or in association with $\Sigma^{0}$ ) from exchange of $S M W / Z / \gamma$ and the latter's KK (i.e., spin1 ) excitations. The new fermions will then decay mostly into SM lepton and Higgs particles (including longitudinal $W / Z$ ) via the Yukawa coupling. Again, these signals look similar to the five-dimensional type I model that we discussed, except that (as mentioned above in the context of comparison to the four-dimensional type III seesaw) if we decouple the gauge KK modes, then there is negligible production of $N$ in association with its $S U(2)_{R}$-partner $\tilde{\ell}$ in the five-dimensional type I case, cf. signal from $\Sigma^{ \pm}-\Sigma^{0}$ via $S M W$ exchange 4in the type III seesaw in five dimensions.

\section{CONCLUSIONS AND OUTLOOK}

Searches have been done (and are ongoing) at the LHC for TeV-mass SM singlet neutrinos involved in the generation of super-small SM neutrino mass via various four-dimensional models of seesaw. However, we have tried to present a case here that many these require a small parameter in order to obtain the right size of the SM neutrino mass, thus in some cases reducing the original attraction of the seesaw. In fact, we feel that there might not be any strong motivation for singlets in these models to be at $\sim \mathrm{TeV}$ other than getting a signal at the LHC from them. In earlier work, some of us had demonstrated that a completely natural realization of $\mathrm{TeV}$-scale seesaw occurs instead in a warped extra-dimensional framework, which is dual (as per the AdS/CFT correspondence) to the SM Higgs being a composite particle arising from some new strong dynamics.

In this paper (and a follow-up), we initiated the study of the LHC phenomenology of this framework of a natural $\mathrm{TeV}$-scale seesaw. In particular, here, we showed that signals similar to the four-dimensional models arise in this warped/composite framework as well. At the same time, the details of the phenomenology are different in an interesting manner. Hence, one can suitably adapt existing searches for singlet neutrinos in four-dimensional models to the natural five-dimensional one.

The easiest way to see how these features arise is using a (effective) two-site picture of this framework. Namely, we have two sectors of the theory: elementary and composite. The SM Higgs is contained in the composite sector, whose characteristic mass scale is $\sim \mathrm{TeV}$ so as to address the Planck-weak hierarchy problem; whereas, the rest of SM particles are admixtures of those in the two sectors, i.e., partially composite/elementary. Specifically, the degree of compositeness of the non-Higgs SM particles reflects the size of their mass, i.e., the top quark is significantly composite, while the light quarks are negligibly so. Moreover, leptonnumber is preserved by the composite sector, but broken at the UV cutoff in the elementary sector. So, if we include an elementary SM singlet right-handed neutrino $\left(N_{R}\right)$, then it will naturally have a super-large, even Planck-scale, Majorana mass. However, by itself, this lepton-number violation is not quite sufficient to induce Majorana mass for $S M$ neutrino, since we also require EWSB/Higgs VEV for this purpose. Thus, this information about lepton-number violation has to be transmitted from the elementary to the composite sector, where the SM Higgs resides. In this way, one can "sew" together the two necessary ingredients in order to generate the SM neutrino mass.

A simple and natural way for sharing lepton-number violation between the two sectors is for the above elementary $N_{R}$ to also mix with composite sector TeV-mass singlets. These singlet states are purely Dirac to begin with, but as a result of the above coupling to elementary $N_{R}$, they acquire a tiny Majorana mass component. It can be shown that it is the exchange of these (now pseudoDirac) singlet states generates-without any tuning-the right size of the SM neutrino mass. Thus, the TeV-mass 
singlets play a crucial role in this entire process: their observation at the LHC would provide a vital test of this mechanism of the SM neutrino mass generation. Just to emphasize, the $\mathrm{TeV}$ mass for these composite singlets is natural, being directly related to the electroweak scale (cf., usual four-dimensional models, where some extra assumptions are typically needed in order to get such a mass for the singlet neutrinos).

The obvious next question is how to produce these TeVmass composite neutrinos $N_{R}$ at the LHC, given that they are SM singlets. The analogous four-dimensional models provide a recipe: typically this is achieved in these models in the context of extending the SM EW symmetry to the left-right $(\mathrm{LR})$ structure, i.e., $S U(2)_{\mathrm{L}} \times S U(2)_{\mathrm{R}} \times U(1)_{\mathrm{B}-\mathrm{L}}$, with $S U(2)_{\mathrm{R}} \times U(1)_{\mathrm{B}-\mathrm{L}}$ broken down to $\mathrm{SM}$ hypercharge at the $\mathrm{TeV}$ scale. The point is that $N_{R}$-while being SM singlet-is a doublet of $S U(2)_{R}$, thus can be produced via decay of charged $W_{R} . W_{R}$ is, in turn, produced via $q \bar{q}$ annihilation with the associated $W_{R}$ couplings of SM EW strength.

Indeed, a similar LR symmetric pattern is motivated in the warped/composite Higgs framework, albeit for a different reason (i.e., than parity restoration in usual four-dimensional models). The purpose of the extra symmetry is to protect $\rho$ parameter from receiving large corrections. So, we assume this extension only in the composite sector as simply a global symmetry. There is then no elementary charged $W_{R}$ gauge boson (unlike for the SM $W_{L}$ ), but we do have composite charged $W_{R}$ 's. However, in this way, it seems naively that we do not have a way to produce $W_{R}$, since the SM quarks inside proton are mostly elementary, leading to a negligible direct coupling to composite-sector $W_{R}$.

Remarkably, we found that elementary-composite $W_{L}$ mixing, followed by composite $W_{L}-W_{R}$ mixing via Higgs VEV, induces the required coupling of composite charged $W_{R}$ 's to quarks. It is the degeneracy among spin-1 composites which ensures that the second mixing effect is rather large for a few $\mathrm{TeV}$ composite $W$ 's. The end result is that coupling of light quarks to $W_{R}$ in these models is suppressed compared to the typical SM EW coupling, but it still sizable. Consequently, although production rates for $W_{R}$ are smaller than in the four-dimensional LR case, as we showed here, it is still enough for discovery. We would like to emphasize here that this subtle effect has been discussed earlier in the context of LHC signals for these spin-1 states in general, i.e., independent of neutrino mass considerations. However, this feature was not really exploited before, in the sense that decay modes of $W_{R}$ studied in that context (for example, $W / Z /$ Higgs) were also accessible via $W_{L}$, i.e., production of $W_{R}$ was not really "needed" (cf. here $N_{R}$ only couples to $W_{R}$ ).

Note that, in the $W_{R}$ decay, the composite $N_{R}$ is accompanied by composite charged lepton, since the associated coupling is, for example, larger than coupling to one composite and one elementary states (cf., in four-dimensonal models, it would be simply the SM charged lepton). Composite charged lepton decays into SM charged lepton, plus Higgs/longitudinal $Z$, while $N_{R}$ decays (just like in fourdimensional models) into SM charged lepton and $W$, latter decaying either leptonically or hadronically. Thus, the final state is either (dilepton $+W$-jet + Higgs $/ Z$ ) or (trilepton + MET + Higgs $/ Z$ ). Note that the dileptons in the first channel are of opposite sign, given the pseudo-Dirac nature of these singlets (cf. same-sign dileptons from Majorana singlets in some four-dimensional LR models).

We performed a detailed analysis of both these channels for singlet neutrino production via decay of composite $W_{R}$, finding that, for both channels, significant evidence can be observed for $\sim 2 \mathrm{TeV} W_{R}$ and composite $N_{R} /$ composite charged lepton of mass $750 \mathrm{GeV}$, with an integrated luminosity of $300 \mathrm{fb}^{-1}$, and even discovery with slightly more integrated luminosity. It is clear that the extra boson in final state permits distinguishing this framework from fourdimensional LR models. In addition, this feature is crucial for reducing the SM background, especially given smaller rate than in four-dimensional LR models and the absence of the "smoking-gun," i.e., same-sign dileptons; indeed, it is noteworthy that in spite of these seeming challenges, we are able to extract a reasonable signal.

Finally, we would like to provide a "preview" of part II, where we will consider signals of singlet neutrinos from production and decay of particles absent in four-dimensional LR models. In particular, one idea is to relax the degeneracy of spin-1 composites that was assumed here. In the light of the above discussion, this direction actually results in suppressing the charged $W_{R}$ signal, but we will show that a "new" type of signal appears from a neutral heavy boson, i.e., which is not accompanied by a charged channel (unlike in the four-dimensional LR case, where charged spin-1 channel is actually dominant, $W_{R}$ being lighter than the corresponding extra neutral gauge boson). We will also study production of composite $S U(2)_{\mathrm{L}}$ doublet leptons inherent to this framework (cf. absent in the four-dimensional LR models); singlet neutrinos can be produced in their decays via a Yukawa coupling, i.e., independent of the couplings of $N_{R}$ to $W_{R}$, thus of the representation of $N_{R}$ under the extended EW symmetry [cf. signals studied earlier do reply on singlet being charged under $\left.S U(2)_{\mathrm{R}} \times U(1)_{\mathrm{B}-\mathrm{L}}\right]$. Overall, our work leads to a new perspective on the nature and relevance of LHC signals of $\mathrm{TeV}$-scale singlet neutrinos.

\section{ACKNOWLEDGMENTS}

We would like to thank Chien-Yi Chen, Roberto Contino, Bhupal Dev, Shrihari Gopalakrishna, and Doojin Kim for discussions and David Curtin for help with simulations. This work was supported in part by National Science Foundation Grant No. PHY-1315155 and the Maryland Center for Fundamental Physics. S. H. was also supported in part by a fellowship from The Kwanjeong Educational Foundation. 


\section{APPENDIX: BRANE LOCALIZED KINETIC TERMS FOR FERMIONS}

In this section, we present details of the effects of IR brane localized kinetic terms (BKT) for the right-handed chiral part of a bulk fermion with $(-+)$, i.e. DirichletNeumann, boundary condition. In particular, we show its effects on the spectrum of KK fermions and the coupling to KK gauge fields. Throughout this section, the fivedimensional metric is taken to be a slice of $\mathrm{AdS}_{5}$

$$
d s^{2}=\left(\frac{z_{h}}{z}\right)^{2}\left(\eta_{\mu \nu} d x^{\mu} d x^{\nu}-d z^{2}\right),
$$

where $\eta_{\mu \nu}=\operatorname{diag}(1,-1,-1,-1)$ and $z$ is the coordinate for the fifth dimension. Also, $z_{h}\left(z_{v}\right)=\frac{1}{k}\left(\frac{e^{k \pi r_{c}}}{k}\right)$ is the position of the UV(IR) brane and $k$ is the AdS curvature. The fivedimensional Lagrangian for a bulk fermion, together with IR BKT for its right-handed chirality, is given by $[17]^{29}$

$$
\begin{aligned}
\mathcal{L}_{5 D \text { Fermion }}= & \frac{i}{2}\left(\bar{\Psi} e_{a}^{M} \Gamma^{a} D_{M} \Psi-D_{M} \bar{\Psi} e_{a}^{M} \Gamma^{a} \Psi\right) \\
& +c k \bar{\Psi} \Psi+\frac{\delta\left(z-z_{v}\right)}{z_{h} / z} \frac{r_{\mathrm{IR}}}{k} \bar{\Psi}_{R} i \gamma^{\mu} \partial_{\mu} \Psi_{R},
\end{aligned}
$$

where $M=0,1,2,3,5\left(x^{5}=z\right), \mu=0,1,2,3$ and $e_{a}^{M}=$ $\left(z / z_{h}\right) \delta_{a}^{M}$ is funfbein. Here, $c$ denotes the bulk Dirac mass of the fermion in unit of AdS curvature $k$ and $r_{\mathrm{IR}}$ is a dimensionless coefficient parametrizing the size of IR BKT. In addition, $D_{\mu}=\partial_{\mu}+\gamma_{\mu} \gamma_{5} /(4 z)$ and $D_{5}=\partial_{5}$. We use projection operator $P_{R}=\left(1+\gamma_{5}\right) / 2$ to define $\Psi_{R}=P_{R} \Psi$. If the fermion is charged under some gauge forces, these covariant derivatives will contain, in addition to spacetime connection, gauge connection as well. In particular, in order to make the whole Lagrangian gauge invariant, we need to add brane gauge interaction as well as bulk interaction.

$\mathcal{L}_{5 D \text { int }}=g \bar{\Psi} e_{a}^{M} \Gamma^{a} A_{M} \Psi+g \frac{\delta\left(z-z_{v}\right)}{z_{h} / z} \frac{r_{\mathrm{IR}}}{k} \bar{\Psi}_{R} \gamma^{\mu} A_{\mu} \Psi_{R}$,

where $A_{M(\mu)}=T^{a} A_{M(\mu)}^{a}$ with $T^{a}$ being the generator of the gauge group and $g$ is the gauge coupling constant. Decomposing the five-dimensional spinor into two, fourdimensional Weyl spinors

$$
\Psi=\left(\begin{array}{c}
\chi \\
\bar{\psi}
\end{array}\right),
$$

five-dimensional action can be written as

$$
\begin{aligned}
S_{5 D} & =S_{5 D \text { Fermion }}+S_{5 D \text { int }} \\
S_{5 D \text { Fermion }} & =\int d^{5} x\left(\frac{z_{h}}{z}\right)^{4}\left\{-\bar{\chi} i \bar{\sigma}^{\mu} \partial_{\mu} \chi-\psi i \sigma^{\mu} \partial_{\mu} \bar{\psi}\left(1+\frac{\delta\left(z-z_{v}\right)}{z_{h} / z} \frac{r_{\mathrm{IR}}}{k}\right)+\frac{1}{2}\left(\psi \overleftrightarrow{\partial}_{5} \chi-\bar{\chi} \overleftrightarrow{\partial}_{5} \bar{\psi}\right)+\frac{c}{z}(\psi \chi+\bar{\chi} \bar{\psi})\right\} \\
S_{5 D \text { int }} & =\int d^{5} x\left(\frac{z_{h}}{z}\right)^{4}\left\{g \bar{\chi} \bar{\sigma}^{\mu} A_{\mu} \chi+g \psi \sigma^{\mu} A_{\mu} \bar{\psi}\left(1+\frac{\delta\left(z-z_{v}\right)}{z_{h} / z} \frac{r_{\mathrm{IR}}}{k}\right)\right\},
\end{aligned}
$$

where $\overleftrightarrow{\partial}_{5}=\overrightarrow{\partial_{5}}-\overleftrightarrow{\partial_{5}}$ with the convention that the derivative acts only on spinors, not on the metric.

\section{Mass spectrum of KK fermions}

The five-dimensional equations of motion for fermions are given by

$$
\begin{gathered}
-i \bar{\sigma}^{\mu} \partial_{\mu} \chi-\partial_{5} \bar{\psi}+\frac{c+2}{z} \bar{\psi}=0 \\
-i \sigma^{\mu} \partial_{\mu} \bar{\psi}\left(1+\delta\left(z-z_{v}\right) z_{v} r_{\mathrm{IR}}\right)+\partial_{5} \chi+\frac{c-2}{z} \chi=0 .
\end{gathered}
$$

We then KK decompose $\chi$ and $\psi$ as

\footnotetext{
${ }^{29}$ Here, we assume no tree level IR BKT for gauge fields. In principle, there will still be the loop-induced IR BKT for gauge fields. Such terms, however, are too small to generate any sizable effects and we neglect them for simplicity.
}

$\chi=\sum_{n} f_{L n}(z) \chi_{n}(x) \quad$ and $\quad \psi=\sum_{n} f_{R n}(z) \psi_{n}(x)$,

where $\chi_{n}$ and $\psi_{n}$ satisfy four-dimensional Dirac equations

$$
-i \bar{\sigma}^{\mu} \partial_{\mu} \chi_{n}+m_{n} \bar{\psi}_{n}=0 \quad \text { and } \quad-i \sigma^{\mu} \partial_{\mu} \bar{\psi}_{n}+m_{n} \chi_{n}=0 .
$$

Using Eqs. (A8) and (A9) into Eq. (A7), the bulk equations of motion (i.e. dropping terms with $\delta$-function) become two coupled first order differential equations for profile functions

$$
\begin{aligned}
& f_{R n}^{\prime}+m_{n} f_{L n}-\frac{c+2}{z} f_{R n}=0 \\
& f_{L n}^{\prime}-m_{n} f_{R n}+\frac{c-2}{z} f_{L n}=0
\end{aligned}
$$

where $f^{\prime}$ denotes $\frac{d f}{d z}$. For $m_{n} \neq 0$, we get two decoupled second-order differential equations: 

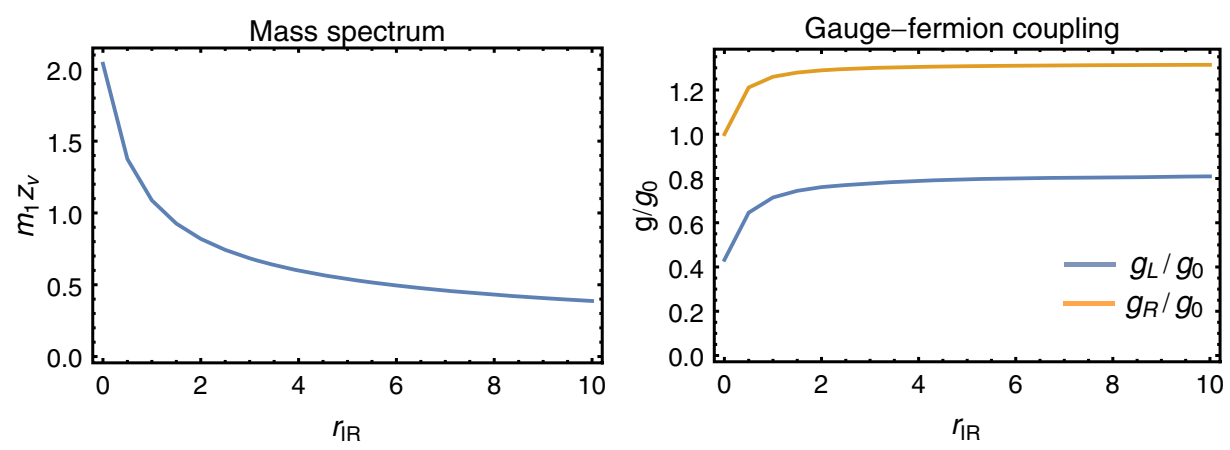

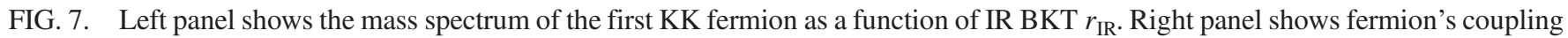
to the first KK gauge field, with (-+) boundary condition for KK gauge boson and right chirality of KK fermion. Coupling to the left (right) chirality of the first KK fermions is denoted as $g_{L(R)}$, where $g_{0}$ is the coupling of right chirality with $r_{\mathrm{IR}}=0$. In both plots, we take $c=-0.27$ and $k \pi r_{c}=34$.

$$
\begin{aligned}
& z^{2} f_{R n}^{\prime \prime}-4 z f_{R n}+\left(m_{n}^{2}-\left(c^{2}-c-6\right)\right) f_{R n}=0 \\
& z^{2} f_{L n}^{\prime \prime}-4 z f_{L n}+\left(m_{n}^{2}-\left(c^{2}+c-6\right)\right) f_{L n}=0
\end{aligned}
$$

These are Bessel equations and the general solutions for $f_{L n}$ and $f_{R n}$ are linear combinations of Bessel functions

$f_{L n}(z)=\left(\frac{z}{z_{h}}\right)^{\frac{5}{2}}\left(A_{n} J_{-c-\frac{1}{2}}\left(m_{n} z\right)+B_{n} Y_{-c-\frac{1}{2}}\left(m_{n} z\right)\right)$

$f_{R n}(z)=\left(\frac{z}{z_{h}}\right)^{\frac{5}{2}}\left(C_{n} J_{-c+\frac{1}{2}}\left(m_{n} z\right)+D_{n} Y_{-c+\frac{1}{2}}\left(m_{n} z\right)\right)$.

The first order differential equations Eq. (A10) provide further constraints on these coefficients

$$
A_{n}=-C_{n} \quad \text { and } \quad B_{n}=-D_{n} .
$$

Thus only two are independent coefficients (call them $b_{n}$ and $N_{n}$, which are formed from $A_{n} \sim D_{n}$ ) and the profiles can be rewritten with three undetermined parameters $m_{n}, b_{n}$ and $N_{n}$ as

$f_{L n}(z)=-\frac{\left(\frac{z}{z_{h}}\right)^{\frac{5}{2}}}{N_{n} \sqrt{\pi r_{c}}}\left(J_{-c-\frac{1}{2}}\left(m_{n} z\right)+b_{n} Y_{-c-\frac{1}{2}}\left(m_{n} z\right)\right)$

$f_{R n}(z)=\frac{\left(\frac{z}{z_{h}}\right)^{\frac{5}{2}}}{N_{n} \sqrt{\pi r_{c}}}\left(J_{-c+\frac{1}{2}}\left(m_{n} z\right)+b_{n} Y_{-c+\frac{1}{2}}\left(m_{n} z\right)\right)$.

To determine these parameters, one needs boundary conditions as well as orthonormal conditions. The consistent boundary conditions for this Lagrangian can be obtained following [17]
$\mathrm{UV}: \psi=0$
$\mathrm{UV}: f_{R n}=0$
$\mathrm{IR}: \chi=-r_{\mathrm{IR}} z_{v} i \sigma^{\mu} \partial_{\mu} \bar{\psi}$
IR: $f_{L n}=-r_{\mathrm{IR}} m_{n} z_{v} f_{R n}$.

(A17)
Notice that this is where the effects of BKT appears first time, eventually leading to changes in spectrum and couplings. Namely, the (bulk) equations of motion we solve is the same with the case without BKT. The differences caused by BKT are encoded in the boundary conditions Eq. (A17). Imposing the boundary conditions Eq. (A17) to the ansatz Eq. (A16), we get

$$
\frac{J_{-c+\frac{1}{2}}\left(m_{n} z_{h}\right)}{Y_{-c+\frac{1}{2}}\left(m_{n} z_{h}\right)}=\frac{J_{-c-\frac{1}{2}}\left(m_{n} z_{v}\right)-r_{\mathrm{IR}} m_{n} z_{v} J_{-c+\frac{1}{2}}\left(m_{n} z_{v}\right)}{Y_{-c-\frac{1}{2}}\left(m_{n} z_{v}\right)-r_{\mathrm{IR}} m_{n} z_{v} Y_{-c+\frac{1}{2}}\left(m_{n} z_{v}\right)}=-b_{n} .
$$

The spectrum of KK fermions $\left(m_{n} z_{v}\right)$ can be solved using the above equation as a function of $r_{\mathrm{IR}}$. As a specific example, we choose $c=-0.27$. This is the value for $N$ and $\tilde{\ell}$ fields we use in earlier sections chosen to satisfy all the phenomenological constraints [2]. The result of the mass of first KK fermions is shown in Fig. 7. In Sec. II of this paper, we demand the mass of $N$ or $\tilde{\ell}$ to be less than half of the mass of $\mathrm{KK}$ gauge bosons $W_{L} / W_{R}$. The first KK gauge bosons with ether $(++)$ or $(-+)$ boundary conditions have mass $m_{n} z_{v} \sim 2.4$. It is clear from Fig. 7 that $O(1) r_{\mathrm{IR}}$ is enough to bring the mass of the first $\mathrm{KK}$ fermions below the half of the mass of KK gauge bosons. A Similar effect in the case of gauge fields, i.e. lowering the KK scale using gauge IR BKT, is studied in [35].

Orthonormal conditions are given by

$$
\begin{aligned}
\int d z\left(\frac{z_{h}}{z}\right)^{4}\left(1+\delta\left(z-z_{v}\right) z_{v} r_{\mathrm{IR}}\right) f_{R n} f_{R_{m}} & =\delta_{n m} \\
\int d z\left(\frac{z_{h}}{z}\right)^{4} f_{L n} f_{L_{m}} & =\delta_{n m}
\end{aligned}
$$

From these we can obtain the expression for $N_{n}$ as 


$$
\begin{aligned}
N_{n}^{2}= & \frac{1}{2 \pi r_{c} z_{h}}\left(1+\left(m_{n} z_{v}\right)^{2} r_{\mathrm{IR}}^{2}+2\left(c+\frac{1}{2}\right) r_{\mathrm{IR}}\right) \\
& \times\left.\left[z^{2}\left(J_{-c+\frac{1}{2}}\left(m_{n} z\right)+b_{n} Y_{-c+\frac{1}{2}}\left(m_{n} z\right)\right)^{2}\right]\right|_{z_{h}} ^{z_{v}} \\
\approx & \frac{z_{v}^{2}}{2 \pi r_{c} z_{h}}\left(1+\left(m_{n} z_{v}\right)^{2} r_{\mathrm{IR}}^{2}+2\left(c+\frac{1}{2}\right) r_{\mathrm{IR}}\right) \\
& \times\left(J_{-c+\frac{1}{2}}\left(m_{n} z_{v}\right)+b_{n} Y_{-c+\frac{1}{2}}\left(m_{n} z_{v}\right)\right)^{2},
\end{aligned}
$$

where we neglect small terms proportional to $z_{h}^{2}$.

\section{Couplings to KK gauge modes}

In this section, we discuss couplings of first KK modes of gauge fields to the first KK modes of fermions $\bar{\chi}_{1} \gamma^{\mu} \chi_{1} A_{\mu}^{(1)}$ and $\bar{\psi}_{1} \gamma^{\mu} \psi_{1} A_{\mu}^{(1)}$. The KK modes of gauge fields with $(-+)$ boundary conditions have the profile $^{30}$ :

$$
f_{g n}(z)=\sqrt{\frac{1}{z_{h}}} \frac{z}{N_{n}}\left(J_{1}\left(m_{n} z\right)+b_{n} Y_{1}\left(m_{n} z\right)\right),
$$

where $N_{n}$ is given as

\footnotetext{
${ }^{30}$ This choice of boundary condition for gauge field is considered here recalling the fact that in our model it is $W_{R}^{(1)}$, which has $(-+)_{\tilde{C}}$ boundary condition, that couples to $\mathrm{KK}$ fermions $N$ and $\tilde{\ell}$.
}

$$
\begin{aligned}
N_{n}^{2}= & \frac{1}{2}\left[z_{v}^{2}\left(J_{1}\left(m_{n} z_{v}\right)+b_{n} Y_{1}\left(m_{n} z_{v}\right)\right)^{2}\right. \\
& \left.-z_{h}^{2}\left(J_{0}\left(m_{n} z_{h}\right)+b_{n} Y_{0}\left(m_{n} z_{h}\right)\right)^{2}\right],
\end{aligned}
$$

and the spectrum of gauge $\mathrm{KK}$ modes and $b_{n}$ can be obtained by solving

$$
\frac{J_{1}\left(m_{n} z_{h}\right)}{Y_{1}\left(m_{n} z_{h}\right)}=\frac{J_{0}\left(m_{n} z_{v}\right)}{Y_{0}\left(m_{n} z_{v}\right)}=-b_{n}
$$

The couplings of KK fermions and KK gauge bosons are obtained via the overlap of profiles:

$$
\begin{aligned}
& g_{L} \propto \int d z\left(\frac{z_{h}}{z}\right)^{4} f_{L 1}^{2} f_{g 1} \\
& g_{R} \propto \int d z\left(\frac{z_{h}}{z}\right)^{4} f_{R 1}^{2} f_{g 1}\left(1+\delta\left(z-z_{v}\right) r_{\mathrm{IR}} z_{v}\right) .
\end{aligned}
$$

We define $g_{0}$ as $g_{R}$ with no BKT $\left(r_{\mathrm{IR}}=0\right)$. The result of $g_{L(R)} / g_{0}$ as a function of $r_{\mathrm{IR}}$ is shown in Fig. 7. When $r_{\mathrm{IR}}=0, f_{L 1}$ vanishes on the IR brane due to its Dirichlet boundary condition on the IR brane, although it is still localized near the IR brane having nonzero overlap with gauge $\mathrm{KK}$ profile. However, $f_{R 1}$ and $f_{g 1}$ are all peaked at IR brane. Therefore, for $r_{\mathrm{IR}}=0, g_{L}$, albeit nonvanishing, is smaller than $g_{R}$. As we turn on nonzero BKT, $r_{\mathrm{IR}} \neq 0, f_{L 1}$ is no longer zero on the IR brane [Eq. (A17)], leading to rapid increase of its overlap with gauge KK. Indeed, as shown in Fig. 7, gauge couplings to both chirality become similar in strength $\left(g_{L} \sim g_{R}\right)$ for large $r_{\mathrm{IR}}$. This justifies setting the same coupling of KK gauge bosons $W_{L} / W_{R}$ to both chirality of $N$ and $\tilde{\ell}$ in Sec. III.
[1] P. Minkowski, Phys. Lett. B 67, 421 (1977); T. Yanagida in Proceedings: Workshop on the Unified Theories and the Baryon Number in the Universe: Tsukuba, Japan, 1979 (1979), p. 95; M. Gell-Mann, P. Ramond, and R. Slansky, Supergravity (North Holland, Amsterdam, 1979), p. 315; S. L. Glashow, in Proceedings of 1979 Cargese Summer Institute on Quarks and Leptons (Plenum, New York, 1980), p. 687; R. N. Mohapatra and G. Senjanovic, Phys. Rev. Lett. 44, 912 (1980).

[2] S. J. Huber and Q. Shafi, Phys. Lett. B 583, 293 (2004); C. Csaki, C. Grojean, J. Hubisz, Y. Shirman, and J. Terning, Phys. Rev. D 70, 015012 (2004); M. Carena, A. D. Medina, N. R. Shah, and C. E. M. Wagner, Phys. Rev. D 79, 096010 (2009).

[3] G. Perez and L. Randall, J. High Energy Phys. 01 (2009) 077; C. Csaki, C. Delaunay, C. Grojean, and Y. Grossman, J. High Energy Phys. 10 (2008) 055.

[4] K. Agashe, S. Hong, and L. Vecchi, Phys. Rev. D 94, 013001 (2016).
[5] R. N. Mohapatra, Phys. Rev. Lett. 56, 561 (1986); R. N. Mohapatra and J. W. F. Valle, Phys. Rev. D 34, 1642 (1986).

[6] M. Malinsky, J. C. Romao, and J. W. F. Valle, Phys. Rev. Lett. 95, 161801 (2005).

[7] For production of singlet neutrinos via $W_{R}^{ \pm}$decays in inverse seesaw models, see, for example, C. Y. Chen and P. S. B. Dev, Phys. Rev. D 85, 093018 (2012); P. S. Bhupal Dev and R. N. Mohapatra, Phys. Rev. Lett. 115, 181803 (2015); F. F. Deppisch, L. Graf, S. Kulkarni, S. Patra, W. Rodejohann, N. Sahu, and U. Sarkar, Phys. Rev. D 93, 013011 (2016); A. Das, N. Nagata, and N. Okada, J. High Energy Phys. 03 (2016) 049.

[8] For production of singlet neutrinos via their mixing with doublet ones in inverse seesaw models, see, for example, F. del Aguila and J. A. Aguilar-Saavedra, Phys. Lett. B 672, 158 (2009); F. del Aguila, J. A. Aguilar-Saavedra, and J. de Blas, Acta Phys. Pol. B 40, 2901 (2009); E. Arganda, M. J. Herrero, X. Marcano, and C. Weiland, Phys. Lett. B 
752, 46 (2016); A. Das and N. Okada, Phys. Rev. D 93, 033003 (2016); A. Das, P. S. Bhupal Dev, and N. Okada, Phys. Lett. B 735, 364 (2014); A. Das and N. Okada, Phys. Rev. D 88, 113001 (2013); T. Saito, M. Asano, K. Fujii, N. Haba, S. Matsumoto, T. Nabeshima, Y. Takubo, H. Yamamoto, and K. Yoshioka, Phys. Rev. D 82, 093004 (2010); N. Haba, S. Matsumoto, and K. Yoshioka, Phys. Lett. B 677, 291 (2009); for NLO corrections, see, for example, A. Das, P. Konar, and S. Majhi, J. High Energy Phys. 06 (2016) 019; A. Das, arXiv:1701.04946; C. Degrande, O. Mattelaer, R. Ruiz, and J. Turner, Phys. Rev. D 94, 053002 (2016); R. E. Ruiz, arXiv:1509.06375.

[9] R. N. Mohapatra, Nucl. Phys. B908, 423 (2016).

[10] R. Contino, T. Kramer, M. Son, and R. Sundrum, J. High Energy Phys. 05 (2007) 074; M. Low, A. Tesi, and L. T. Wang, Phys. Rev. D 92, 085019 (2015).

[11] K. Agashe, P. Du, and S. Hong, following article, Phys. Rev. D 97, 075033 (2018).

[12] K. Agashe, H. Davoudiasl, S. Gopalakrishna, T. Han, G. Y. Huang, G. Perez, Z. G. Si, and A. Soni, Phys. Rev. D 76, 115015 (2007).

[13] K. Agashe, S. Gopalakrishna, T. Han, G. Y. Huang, and A. Soni, Phys. Rev. D 80, 075007 (2009).

[14] K. Agashe, A. Delgado, M. J. May, and R. Sundrum, J. High Energy Phys. 08 (2003) 050.

[15] K. Agashe, R. Contino, L. Da Rold, and A. Pomarol, Phys. Lett. B 641, 62 (2006).

[16] C. Csaki, A. Falkowski, and A. Weiler, J. High Energy Phys. 09 (2008) 008; M. Blanke, A. J. Buras, B. Duling, S. Gori, and A. Weiler, J. High Energy Phys. 03 (2009) 001; M. Bauer, S. Casagrande, U. Haisch, and M. Neubert, J. High Energy Phys. 09 (2010) 017; B. Keren-Zur, P. Lodone, M. Nardecchia, D. Pappadopulo, R. Rattazzi, and L. Vecchi, Nucl. Phys. B867, 394 (2013).

[17] M. Carena, A. Delgado, E. Ponton, T. M. P. Tait, and C. E. M. Wagner, Phys. Rev. D 71, 015010 (2005); C. Csaki, C. Grojean, J. Hubisz, Y. Shirman, and J. Terning, Phys. Rev. D 70, 015012 (2004).

[18] R. Contino and M. Salvarezza, J. High Energy Phys. 07 (2015) 065.
[19] The ATLAS Collaboration, Report No. ATLAS-CONF2016-062; M. Aaboud et al. (ATLAS Collaboration), Phys. Lett. B 765, 32 (2017); The ATLAS Collaboration, Report No. ATLAS-CONF-2016-083.

[20] CMS Collaboration, Report No. CMS-PAS-B2G-16-007.

[21] F. del Aguila, J. de Blas, and M. Perez-Victoria, Phys. Rev. D 78, 013010 (2008); A. Atre, T. Han, S. Pascoli, and B. Zhang, J. High Energy Phys. 05 (2009) 030; S. Antusch and O. Fischer, J. High Energy Phys. 10 (2014) 094.

[22] A. Alloul, N. D. Christensen, C. Degrande, C. Duhr, and B. Fuks, Comput. Phys. Commun. 185, 2250 (2014).

[23] D. Pappadopulo, A. Thamm, R. Torre, and A. Wulzer, J. High Energy Phys. 09 (2014) 060.

[24] J. Alwall, R. Frederix, S. Frixione, V. Hirschi, F. Maltoni, O. Mattelaer, H.-S. Shao, T. Stelzer, P. Torrielli, and M. Zaro, J. High Energy Phys. 07 (2014) 079.

[25] R. D. Ball et al., Nucl. Phys. B867, 244 (2013).

[26] T. Sjostrand, S. Mrenna, and P. Z. Skands, J. High Energy Phys. 05 (2006) 026.

[27] J. de Favereau, C. Delaere, P. Demin, A. Giammanco, V. Lemaître, A. Mertens, and M. Selvaggi (DELPHES 3 Collaboration), J. High Energy Phys. 02 (2014) 057.

[28] M. Cacciari and G. P. Salam, Phys. Lett. B 641, 57 (2006).

[29] M. Cacciari, G. P. Salam, and G. Soyez, Eur. Phys. J. C 72, 1896 (2012).

[30] G. Aad et al. (ATLAS Collaboration), J. Instrum. 11, P04008 (2016).

[31] I. R. Tomalin (CMS Collaboration), J. Phys. Conf. Ser. 110, 092033 (2008).

[32] C. Y. Chen, P. S. B. Dev, and R. N. Mohapatra, Phys. Rev. D 88, 033014 (2013); P. S. B. Dev, D. Kim, and R. N. Mohapatra, J. High Energy Phys. 01 (2016) 118.

[33] D. J. Miller, P. Osland, and A. R. Raklev, J. High Energy Phys. 03 (2006) 034.

[34] T. Han, I. W. Kim, and J. Song, Phys. Lett. B 693, 575 (2010).

[35] For warped models, see, for example, M. Carena, E. Ponton, T. M. P. Tait, and C. E. M. Wagner, Phys. Rev. D 67, 096006 (2003); H. Davoudiasl, J. L. Hewett, and T. G. Rizzo, Phys. Rev. D 68, 045002 (2003). 